\title{
Dr. Right and Dr. Wrong: Zum Einfluss des Doktortitels auf den Wahlerfolg von Direktkandidaten bei der Bundestagswahl 2009'
}

\author{
Sebastian Schneider / Markus Tepe
}

\section{Dr. Right and Dr. Wrong: The Impact of a PhD Title on the Electoral Success of Direct Candi- dates in the German Federal Election 2009}

Abstract: This study explores the extent to which voters use candidates' PhD title as a heuristic to reduce the effort of voting. Besides the direct effect of a PhD title on candidates' electoral results, we explore four contexts that might shape the importance of the $\mathrm{PhD}$ heuristic. Estimation results on the basis of direct candidates in the German Federal Election 2009 confirm that, on average, a $\mathrm{PhD}$ title is associated with a vote-plus of 0.51 percentage points. The strength of this relationship increases if candidates' chances of winning increase and it decreases if the average number of candidates in the constituency holding a $\mathrm{PhD}$ increases. The predicted electoral results for the district with the candidate that assumed a false $\mathrm{PhD}$ title show that the $\mathrm{PhD}$ title was not decisive. Nevertheless, these findings can be used to reconsider how scrutiny proceedings in federal elections should deal with candidates holding a false $\mathrm{PhD}$ title.

Keywords: Direct Candidate, Information Shortcut, German Federal Election 2009, PhD Title, Regression Analysis, Apolitical Features of Electoral Candidates

Schlagwörter: Direktkandidat, Heuristik, Bundestagswahl 2009, Doktortitel, Regressionsanalyse, unpolitische Kandidateneigenschaften

\section{Einleitung}

In den Medien sticht ein mitunter kurioser Aspekt des politischen Wettbewerbs ins Auge: Politikerinnen und Politiker auf Kommunal-, Landes- oder Bundesebene geraten in die Schlagzeilen, weil sie zu Unrecht einen Doktortitel tragen. ${ }^{2}$ In diesen Fällen brachten nicht die üblichen Anstrengungen in Forschung (und Lehre) den gewünschten Titel, sondern Plagiarismus oder der Griff ins Portemonnaie. In wahlrechtlicher Hinsicht ist der Fall des CDU-Direktkandidaten im Wahlkreis Steinfurt III von besonderem Interesse. ${ }^{3}$ Der Bewerber hatte seinen Doktortitel an

1 Danksagung: Wir bedanken uns bei den MitarbeiterInnen des Bundeswahlleiters für die freundliche Unterstützung bei der Zusammenstellung des Datensatzes. Für besonders wertvolle Hinweise und Anregungen bedanken wir uns bei zwei anonymen Gutachtern, der PVS-Redaktion, Bernhard Kittel und Achim Goerres. Der Datensatz und die Analysesyntax stehen für Replikationszwecke zur Verfügung.

2 Aus Gründen sprachlicher Einfachheit werden im Folgenden nur männliche Bezeichnungen verwendet. Diese sind geschlechtsneutral zu verstehen.

3 Weitere prominente und weniger prominente Fälle, in denen Politiker zu Unrecht einen Doktortitel trugen, sind unter anderem in der Frankfurter Allgemeine Zeitung (2011), dem Spiegel (2009), der Berliner Morgenpost (2008) oder dem Merkur (2010) dokumentiert. 
einer Schweizer Universität erworben, einen Titel, der in Deutschland nicht offiziell geführt werden darf. Der Kandidat gewann das Mandat mit einer knappen Mehrheit von 1,9 Prozentpunkten gegenüber dem bisherigen Mandatsträger, einem Sozialdemokraten mit rechtmäßigem Doktortitel (Süddeutsche Zeitung 2010). Bei der Bundestagswahl 2009 lag die durchschnittliche Stimmendifferenz zwischen erst- und zweitplatziertem Direktkandidaten bei 6,7 Prozentpunkten. ${ }^{4}$ Nachdem der ,falsche“ Doktor entdeckt wurde, stellte sich die Frage, ob die Angabe des Titels auf dem amtlichen Wahlzettel den wahlentscheidenden Vorteil verschafft hatte. Der Einspruch auf nachträgliche Prüfung eines Wahlmangels gem. \$ 2 Abs. 4 Satz 2 WPrüfG wurde jedoch vom Bundestagspräsidenten abgelehnt, weil es als „eher unwahrscheinlich“ angesehen wurde, dass der Doktortitel "grundsätzlich geeignet“ ist, einen entscheidenden Vorteil gegenüber anderen Wahlbewerbern herzustellen (Bundestag 2009, S. 1-2). Dieser Beitrag möchte den Vorfall zum Anlass nehmen, um eine fundierte, empirische Prüfung des Zusammenhangs zwischen dem Doktortitel und dem Wahlerfolg von Direktkandidaten zu liefern.

Seit längerem ist bekannt, dass unpolitische Kandidateneigenschaften, wie z. B. das Alter, das Geschlecht oder die physische Attraktivität, Einfluss auf den Wahlerfolg der Direktkandidaten nehmen (Kelley u. McAllister 1984; McDermott 1997; Mackenrodt 2008; Rosar 2009). Die Befunde werden damit erklärt, dass die Beschaffung und Verarbeitung von Informationen zur Qualität der Kandidaten für den Wähler Kosten verursacht und Zeit beansprucht. Die Wähler greifen daher auf schnell und kostenlos verfügbare Informationshilfen (sog. Heuristiken) zurück, um die politischen Kandidateneigenschaften näherungsweise zu erfassen (Steenbergen 2010, S. 13; Popkin 1993). Der Doktortitel kann ebenfalls die Funktion einer Entscheidungsheuristik übernehmen. Im Vergleich zu originär politischen Kandidateneigenschaften wie der Parteizugehörigkeit sollte der Einfluss des Doktortitels auf den Wahlerfolg wohl eher gering ausfallen. Allerdings könnte sich dies in dem Maße geändert haben, in dem unpolitische Kandidateneigenschaften durch Personalisierungs- und Individualisierungstendenzen im deutschen Wahlsystem an Bedeutung gewinnen (Wattenberg 1991; Kaase 1994; Lass 1995; Pappi u. Shikano 2001; Brettschneider 2002; Zittel u. Gschwend 2007). Neben dem direkten Einfluss des Doktortitels sollen daher vier Kontexte vorgestellt werden, die Art und Stärke des Zusammenhangs beeinflussen könnten: der Doktorgrad, die Gewinnchance und Parteizugehhörigkeit des Kandidaten sowie die Anzahl der Kandidaten im Wahlkreis, die ebenfalls einen Doktortitel führen. Auf Basis der tatsächlichen Wahlergebnisse der Bundestagswahl 2009 werden drei Fragen untersucht (Bundeswahlleiter 2009): Erstens, welchen Einfluss hat der Doktortitel auf den Erststimmenanteil eines Direktkandidaten? Zweitens, wie beeinflussen Kontextfaktoren die Wirkung des Doktortitels? Drittens, wie wahrscheinlich ist es, dass der ,falsche“ Doktortitel im Wahlkreis Steinfurt III wahlentscheidend war?

4 Den deutlichsten Stimmenvorsprung erzielte Karl-Theodor zu Guttenberg (CSU) mit 53,4 Prozentpunkten gegenüber dem Zweitplazierten. Den geringsten Stimmenvorsprung erzielte Brigitte Zypries (SPD) mit 0,03 Prozentpunkten gegenüber dem Zweitplazierten. 
Der Artikel gliedert sich wie folgt: In Abschnitt 2 wird der Doktortitel-Effekt theoretisch begründet, die Befunde bisheriger Arbeiten zum Einfluss des Doktortitels auf den Wahlerfolg zusammengefasst und die Argumente für die Kontextualisierung des Doktortitel-Effektes hergeleitet. In Abschnitt 3 werden die Datenbasis, Operationalisierung und Analysestrategie vorgestellt. Die Befunde der Datenanalyse werden in Abschnitt 4 präsentiert. Abschnitt 5 fasst die Ergebnisse zusammen und diskutiert, welche Schlussfolgerungen aus der Analyse gezogen werden können.

\section{Theoretischer Rahmen}

\subsection{Der Doktortitel-Effekt}

Aus der politisch-psychologischen Wahlforschung ist bekannt, dass Wähler durch den Einsatz von Heuristiken versuchen, die Komplexität und den Aufwand der Wahlentscheidung zu reduzieren (Steenbergen 2010, S. 17; Faas et al. 2010). Für den Einsatz des Doktortitels als Entscheidungsheuristik dürfte die öffentliche Signalwirkung des Titels eine wichtige Rolle übernehmen. Nach Spence $(1973,2002)$ dienen Titel dazu, Vertrauen zwischen einander unbekannten Akteuren herzustellen. In diesem Punkt kann die Wahlsituation mit einem Bewerbungsverfahren verglichen werden. Der Wähler weiß nur wenig über den Aspiranten und vertraut daher auf standardisierte Leistungsnachweise. Unter Unsicherheit und Informationsmangel wird der Doktortitel dazu genutzt, die entscheidungsrelevanten politischen Kandidateneigenschaften näherungsweise zu bestimmen (Popkin 1993). Mit dem Doktortitel wird in der Regel nicht nur fachliche Kompetenz in Verbindung gebracht, sondern auch anerkannte Tugenden wie Leistungsbereitschaft, Sorgfalt und Glaubwürdigkeit. Letzteres dürfte im politischen Wettbewerb von größter Bedeutung sein. ${ }^{5}$ Diese Erklärung für einen positiven Zusammenhang zwischen Doktortitel und Wahlerfolg eines Direktkandidaten fokussiert auf den Wähler, der den Doktortitel auf dem Stimmzettel sieht und sich entsprechend entscheidet.

Eine zweite Erklärung für den Zusammenhang zwischen Doktortitel und Wahlerfolg könnte darin bestehen, dass Personen mit einem Doktortitel in der politischen Kommunikation vor der Wahl systematisch bevorzugt werden. Die mediale Darstellung von Politikern kann einen erheblichen Einfluss darauf haben, wie Wähler politische Kandidaten wahrnehmen und für wen sie ihre Stimme abgeben (Maurer u. Schoen 2010, S. 278). Wenn Journalisten bei der Berichterstattung denselben Heuristiken unterliegen würden wie andere Menschen, würde über Kandidaten mit Doktortitel häufiger und positiver berichtet als über Bewerber ohne Titel. Im Ergebnis würden sich die Wähler überzufällig für den Kandidaten mit Doktortitel entscheiden, ohne dass sie den Doktortitel überhaupt bewusst zur Kenntnis genommen hätten. Analoge Effekte konnten bereits hinsichtlich der Wir-

5 Ein anekdotischer Beleg für diese Einschätzung findet sich beispielsweise in der Biografie von Guido Westerwelle (Sattar 2009, S. 58), in der Westerwelles Vater mit den Worten zitiert wird: „Junge, mach den Doktor. Dann kannste noch so großen Unsinn reden - jeder wird dir glauben“. 
kung der physischen Attraktivität des Direktkandidaten auf die Berichterstattung bestätigt werden. Maurer und Schoen (2010, S. 291) konnten zeigen, dass über attraktive Wahlkreiskandidaten deutlich häufiger und deutlich positiver berichtet wird als über unattraktive.

Obwohl beiden Erklärungen unterschiedliche Annahmen über das individuelle Wahlverhalten zugrunde liegen, legen beide den Schluss nahe, dass der Doktortitel den Wahlerfolg eines Direktkandidaten positiv beeinflusst. Konkret handelt sich um einen intra-individuellen Stimmenzugewinn, d. h. der Doktortitel führt zu einer Verbesserung gegenüber dem Ergebnis, das derselbe Kandidat (kontrafaktisch) unter denselben Umständen ohne Doktortitel erzielt hätte. Welche Erklärung letztendlich für den positiven Zusammenhang zwischen Doktortitel und Wahlerfolg ursächlich ist, kann im Rahmen dieses Beitrags nicht unterschieden werden. Die allgemeine Ausgangshypothese lautet daher:

\section{H1: Der Doktortitel erhöht den Stimmenanteil eines Direktkandidaten.}

Bisherige Studien zum Zusammenhang zwischen Doktortitel und Wahlerfolg kommen zu keinem eindeutigen Ergebnis. Ohne Anspruch auf Vollständigkeit liefert Tabelle 1 eine Zusammenfassung der empirischen Befunde zum DoktortitelEffekt aus den vergangenen 20 Jahren. Bei Bundestagswahlen erhöht ein Doktortitel den durchschnittlichen Erststimmenanteil um 0,42 bis 0,72 Prozentpunkte. Allerdings ist der Effekt nur bei Klein und Rosar (2005) statistisch signifikant, bei Mackenrodt (2008) hingegen nicht. Die Studien von Faas und Schoen (2006) und Rosar, Klein und Beckers (2008) zu Landtagswahlen bestätigen einen positiven Zusammenhang zwischen Doktortitel und Erststimmenanteil, weisen jedoch keine p-Werte aus. Auch länderspezifische Unterschiede scheinen die Signalwirkung des Doktortitels zu beeinflussen. Die Studie von Kelley und McAllister (1984) legt nahe, dass der Doktortitel negativ konnotiert sein könnte. Im eher klassenbasierten Parteienwettbewerb Großbritanniens scheinen sich hohe formale Bildungsabschlüsse negativ auf den Wahlerfolg des Kandidaten auszuwirken, wobei der Effekt nicht statistisch signifikant ist. 
Tabelle 1: Bisherige Befunde zum Doktortitel-Effekt

\begin{tabular}{|c|c|c|c|c|c|}
\hline Autor(en) & Jahr & Ebene & $\begin{array}{l}\text { Effekt } \\
\text { (p-Wert) }\end{array}$ & $\begin{array}{l}\text { Abhängige Va- } \\
\text { riable }\end{array}$ & Kontrollvariablen \\
\hline Rosar (2009) & $\begin{array}{l}1990- \\
2008\end{array}$ & Bundesländer & $\begin{array}{l}2,02-5,32 \\
(\min . \leq \\
0,05)\end{array}$ & $\begin{array}{l}\text { Zweitstimmen } \\
\text { Partei }\end{array}$ & $\begin{array}{l}\text { Persönliche Eigen- } \\
\text { schaften, politische } \\
\text { Erfahrung, phys. } \\
\text { Attraktivität, Alter, } \\
\text { Geschlecht }\end{array}$ \\
\hline $\begin{array}{l}\text { Rosar, Klein } \\
\text { u. Beckers } \\
(2008)\end{array}$ & 2005 & $\begin{array}{l}\text { Nordrhein-West- } \\
\text { falen }\end{array}$ & $\begin{array}{l}0,18 \\
\text { (n. a.) }\end{array}$ & $\begin{array}{l}\text { Abweichung } \\
\text { vom Stimmen- } \\
\text { anteil der Par- } \\
\text { tei }\end{array}$ & $\begin{array}{l}\text { Phys. Attraktivität, } \\
\text { Geschlecht, Alter, } \\
\text { Immigrant, MdL, } \\
\text { Prominentenstatus, }\end{array}$ \\
\hline $\begin{array}{l}\text { Mackenrodt } \\
(2008)\end{array}$ & $\begin{array}{l}1998- \\
2005\end{array}$ & Deutschland & $\begin{array}{l}0,42 \\
\text { (n. s.) }\end{array}$ & $\begin{array}{l}\text { Erststimmen- } \\
\text { anteil }\end{array}$ & $\begin{array}{l}\text { Alter, MdB, Ge- } \\
\text { schlecht, Direktkan- } \\
\text { didat Prominenz, } \\
\text { Ausschussmitglied, } \\
\text { Zweitstimmen }\end{array}$ \\
\hline $\begin{array}{l}\text { Faas u. } \\
\text { Schoen } \\
(2006)\end{array}$ & 2003 & Bayern & $\begin{array}{l}0,02 \\
\text { (n. a.) }\end{array}$ & $\begin{array}{l}\text { Erststimmen- } \\
\text { anteil }\end{array}$ & $\begin{array}{l}\text { Platzierung Wahl- } \\
\text { zettel, Geschlecht, } \\
\text { versch. Funktionen } \\
\text { innerhalb der Partei }\end{array}$ \\
\hline $\begin{array}{l}\text { Klein u. } \\
\text { Rosar (2005) }\end{array}$ & 2002 & $\begin{array}{l}\text { National } \\
\text { (Deutschland) }\end{array}$ & $\begin{array}{l}0,72 \\
(\leq 0,05)\end{array}$ & $\begin{array}{l}\text { Erststimmen- } \\
\text { anteil }\end{array}$ & $\begin{array}{l}\text { Attraktivität, Ge- } \\
\text { schlecht, Alter, Par- } \\
\text { tei, Zweitstimmen, } \\
\text { MdB, Ost/West }\end{array}$ \\
\hline $\begin{array}{l}\text { Kelley u. } \\
\text { McAllister } \\
\text { (1984) }\end{array}$ & $\begin{array}{l}1974- \\
1980\end{array}$ & Großbritannien & $\begin{array}{l}-2,2 \\
\text { (n. s.) }\end{array}$ & Stimmenanteil & $\begin{array}{l}\text { Alphabetische Posi- } \\
\text { tion, Geschlecht, } \\
\text { Titel }\end{array}$ \\
\hline
\end{tabular}

Anmerkung: Kelley/McAllister (1984) und Rosar et al. (2008) analysieren den Effekt aller akademischen Abschlüsse. n. a. $=$ nicht angegeben, n. s. $=$ nicht signifikant.

Bereits der Umstand, dass bisherige Arbeiten keine eindeutige Aussage zum $\mathrm{Zu}$ sammenhang zwischen Doktortitel und Wahlerfolg zulassen, spricht für eine weitere empirische Prüfung. Zudem soll die Ablehnung des Einspruchs auf nachträgliche Prüfung eines Wahlmangels gem. $\mathbb{S} 2$ Abs. 4 Satz 2 WPrüfG (Bundestag 2009) im Fall des ,falschen` Doktors empirisch geprüft werden. Die Begründung der Ablehnung stützt sich auf den Vorrang des Bestandsschutzes des einmal gewählten Parlaments und Plausibilitätserwägungen zur Wirkung des Doktortitels (Bundestag 2009, S. 2-3). Für die empirische Fundierung der Ablehnung ist entscheidend, wann ein Mittel „grundsätzlich geeignet“ ist „wahlentscheidend zu sein“. Eine Auslegung bestünde darin, das Argument in zwei Kriterien zu zerlegen. Das Mittel - der Doktortitel - müsste demnach erstens geeignet sein. Dies wäre der Fall, wenn der Effekt des Doktortitels nicht zufällig ist, d. h. der Effekt müsste statistisch signifikant sein (p-Werte). Zweitens müsste das Mittel den wahlentscheidenden Vorteil verursacht haben. Ob der Doktortitel wahlentscheidend war, hängt von den tatsächlichen Stimmenverhältnissen im betroffenen Wahlkreis ab. Diese Sichtweise stellt vergleichsweise hohe Anforderungen an die Begründetheit des nachträglichen Einspruchs gem. $\mathbb{2}$ Abs. 4 Satz 2 WPrüfG. Wird die Aussage, „grundsätzlich geeignet wahlentscheidend zu sein“, stattdessen als ein einziges Kriterium aufgefasst, sinken die Anforderungen. Die Direktkandi- 
daten werden in Einerwahlkreisen nach der Entscheidungsregel der einfachen Mehrheit gewählt. Um ein Direktmandat zu gewinnen, kann bereits eine einzige Stimme wahlentscheidend sein. In dieser Auslegung ist der Doktortitel bereits dann „grundsätzlich geeignet wahlentscheidend zu sein“, wenn gezeigt werden kann, dass der Doktortitel einen positiven, statistisch signifikanten Einfluss auf das Wahlergebnis hat. Ob der Doktortitel im konkreten Fall tatsächlich wahlentscheidend war, ist dabei unerheblich. In dieser Auslegung wird auf das Potenzial des Mittels abgestellt, der realisierte Effekt tritt zunächst in den Hintergrund. Vor dem Hintergrund dieser beiden Auslegungen werden der substanzielle Effekt ( $\beta$-Koeffizient) und die statistische Signifikanz (p-Wert) des Doktortitels geschätzt, um anschließend die Wahrscheinlichkeit zu bestimmen, mit der der ,falsche“ Doktortitel im Wahlkreis Steinfurt III wahlentscheidend war.

\subsection{Kontextualisierung des Zusammenhangs zwischen Doktortitel und Wahlerfolg}

Bisherige Arbeiten zum Doktortitel-Effekt haben den direkten Einfluss des Titels auf den Wahlerfolg untersucht. Dabei scheint es wenig plausibel, dass der Effekt auf den Stimmenanteil unter allen Umständen identisch ist. Exemplarisch werden vier Kontexte vorgestellt, die Art und Stärke des Zusammenhangs zwischen Doktortitel und Wahlerfolg beeinflussen könnten.

Doktorgradspezifischer Doktortitel-Effekt: Der Doktortitel wird üblicherweise von der Fakultät verliehen, weshalb sich Doktorgrade gemäß ihrer Fachbereichszugehörigkeit unterscheiden. Aus der soziologischen Prestigeforschung ist bekannt, dass das Berufsprestige ein wichtiges Merkmal für die soziale Statusgruppenzugehörigkeit ist (Wegener 1992). Allgemein ergibt sich der soziale Status einer Person aus dem durch Symbole kommunizierten sozialen Prestige (Kleining 1975, S. 273). Berufsprestige bezeichnet dabei das Ansehen, das einer beruflichen Tätigkeit und einer beruflichen Position oder Stellung zugeschrieben wird (Hoffmeyer-Zlotnik u. Geis 2003, S. 125-126). Laut der Allensbacher BerufsprestigeSkala (2008) genießen Ärzte das höchste Berufsprestige und das mit großem Abstand gegenüber anderen Berufen. Auf dem amtlichen Wahlzettel ist nicht nur der Titel, sondern auch der Beruf des Kandidaten angegeben. Aus beiden Informationen kann der Wähler ableiten, in welchem Berufsfeld der Kandidat wahrscheinlich promoviert ist. Der Doktortitel-Effekt für einen Dr. med. wäre demnach größer als für einen Dr. jur. oder Dr. rer. nat. Allerdings ist die Zuordnung des Doktortitels zu einem Berufsfeld nicht eindeutig, sodass die Ergebnisse der Allensbacher Berufsprestige-Skala 2008 allenfalls als Näherungsgröße für doktorgradspezifisches Prestige herangezogen werden können.

Studien zur sozialen Zusammensetzung des Deutschen Bundestages liefern eine alternative Erklärung für doktorgradspezifische Effekte. Es wurde wiederholt beobachtet, dass politiknahe Berufe in der Rechtswissenschaft und im öffentlichen Dienst im Bundestag überrepräsentiert sind (Kaack 1981, S. 185, 1988). In Großbritannien und den USA konnte im Zeitverlauf eine Reduzierung der beruflichen Vielfältigkeit im Parlament beobachtet werden, die als Hinweis auf die Professionalisierung des Parlaments gewertet wurde (Rosenthal 1981; Squire 1992; Riddel 1993). Analog zu McDermott (2005), die die Politiknähe der beruflichen Tätig- 
keit des Kandidaten als Entscheidungsheuristik einführt, könnte die Politiknähe des Doktorgrades einen doktorgradspezifischen Doktortitel-Effekt hervorrufen. Wähler würden die Politiknähe des Doktorgrades als Informationshilfe für die Beurteilung von politischen Fähigkeiten nutzen. Dieser Effekt könnte durch den über die Medienberichterstattung laufenden Wirkungspfad verstärkt werden, wenn Journalisten den Doktorgrad bei der Berichterstattung selektiv berücksichtigen (d. h., der Dr. jur. wird zum Rechtsexperten stilisiert, der Dr. rer. pol. zum Wirtschaftsexperten etc.). In diesem Fall könnte ein Doktorgrad in den politiknahen Fachbereichen Rechts-, Sozial- oder Wirtschaftswissenschaften mit besonders hohen Stimmengewinnen in Verbindung gebracht werden.

Beide Erklärungsansätze treffen unterschiedliche Annahmen über die Perzeption des Doktorgrades und liefern damit konträre Vorhersagen über die Wirkung des Doktorgrades. Vor diesem Hintergrund kann keine gerichtete Hypothese formuliert werden. ${ }^{6}$ Stattdessen wird der doktorgradspezifische Effekt wie folgt beschrieben:

H2: Der positive Effekt des Doktortitels auf den Stimmenanteil eines Direktkandidaten variiert mit dem Doktorgrad.

Doktortitel-Effekt und Gewinnchance: Aus der Kandidatenperspektive könnte der Doktortitel gezielt als Bestandteil einer Individualisierungsstrategie eingesetzt werden. Zittel und Gschwend (2007) beobachten eine Tendenz zur Individualisierung des Wahlkampfes der Direktkandidaten. Diese lösen sich zunehmend von den Vorgaben und Themen ihrer Partei und begreifen sich als „eigenständiges politisches Programm“. Zittel und Gschwend (2007, S. 298) vermuten, dass die Intensität der Anreize zur Individualisierung maßgeblich von den Gewinnchancen im Wahlreis abhängt. Die empirischen Befunde auf Basis der Deutschen Kandidatenstudie bestätigen die Vermutung. Kandidaten, die sich hohe Gewinnchancen ausrechnen, neigen zu besonders weitgehenden Formen der individualisierten Wahlkampfführung (Zittel u. Gschwend 2007, S. 315).

Nun stellt sich die Frage, ob eine Individualisierungsstrategie die auf den Doktortitel setzt aufgeht. Das heißt, nutzen die Wähler das Signal Doktortitel nur dann als Entscheidungsheuristik, wenn die Gewinnchance des Bewerbers hoch ist? Diese Frage lässt sich mit den tatsächlichen Wahlergebnisdaten des Bundeswahlleiters überprüfen. Im Gegensatz zur Zweitstimme (Verhältniswahl), zählt bei der Wahl des Direktkandidaten (einfache Mehrheitswahl) nur, welcher Kandidat die meisten Stimmen auf sich vereinigt. Das führt dazu, dass nur eine Stimme für einen der beiden aussichtsreichsten Kandidaten über den Wahlausgang entscheidet (Herrmann 2010, S. 667). Unter der Annahme, dass sich die Wähler dieser Konsequenz des Wahlverfahrens bewusst sind, werden sie ihre Erststimmen nicht vergeuden, sondern strategisch einsetzen, „um dem ,kleineren Übel' unter den beiden aussichtsreichsten Kandidaten zum Sieg zu verhelfen“ (Herrmann

6 Im engeren methodischen Sinne schildert die Hypothese keinen konditionalen Effekt, sondern eine Präzisierung des Doktortitel-Effekts. 
2010, S. 667). ${ }^{7}$ Bezogen auf den Doktortitel-Effekt würde ein solches individuelles Wahlverhalten bedeuteten, dass der positive Einfluss des Doktortitels auf den Wahlerfolg des Kandidaten unerheblich ist, wenn die Gewinnchance des Bewerbers gering ist. Zugespitzt stelle man sich den fiktiven Fall eines Kandidaten der Partei Die Linke vor, der in einem von der CSU dominierten Wahlkreis antritt. Der beschriebene Zusammenhang zwischen Gewinnchance und Doktortitel-Effekt lässt vermuten, dass der Bewerber keinen substanziellen Stimmenzuwachs aus einem Doktortitel generieren kann, weil dem Wähler die Bewerbung chancenlos erscheint.

Zugleich könnte vor dem Hintergrund veränderter „Wählermärkte“ (Zittel u. Gschwend 2007, S. 269) der Stellenwert des Doktortitels als Entscheidungsheuristik zunehmen, je geringer die Differenz zwischen den Gewinnchancen der beiden aussichtsreichsten Kandidaten ist. Wenn eine geringe Differenz der Gewinnchancen der beiden aussichtsreichsten Kandidaten das Ergebnis schwacher Parteienbindung ist, bestünde die Möglichkeit, dass sich Wähler mit schwachen parteipolitischen Affinitäten in ihrer Stimmabgabe stärker von unpolitischen Kandidateneigenschaften wie dem Doktortitel leiten lassen. Beide Erklärungen weisen in die selbe Richtung und werden in folgender Hypothese verdichtet:

\section{H3: Je geringer die Gewinnchance des Kandidaten, desto kleiner ist der Effekt des Doktortitels auf den Stimmenanteil eines Direktkandidaten.}

Doktortitel-Effekt und Parteizugehörigkeit: Welchen Einfluss hat die Parteizugehörigkeit des Kandidaten auf die Wirkung des Doktortitels? Könnte es sein, dass der Doktortitel-Effekt von der Parteizugehörigkeit des Kandidaten moderiert wird? In der statistischen Analyse wird das Zweitstimmenergebnis der Partei des Bewerbers berücksichtigt (siehe 2.3), weshalb die Stimmen, die für einen solchen Effekt verantwortlich sind, von Wählern ausgehen müssten, die ihre Zweitstimme einer anderen Partei gegeben haben. Die empirische Analyse verwendet Aggregatsdaten. Aus einer hohen Korrelation zwischen Erst- und Zweitstimmenanteil kann nicht ohne Weiteres gefolgert werden, dass die Wähler dieselbe Partei gewählt haben (Gschwend u. Pappi 2004, S. 171). Das entspräche einem ökologischen Fehlschluss (vgl. z. B. Bryman 2008, S. 306-307). Offizielle Statistiken zum Splittingphänomen legen nahe, dass das straight ticket weiterhin die empirisch dominierende Wahlentscheidung ist (Johann 2009, S. 399). Wie verhalten sich aber Wähler, die sich für das ticket splitting entschieden haben? Werden sie in Ihrer Wahlentscheidung vom Doktortitel beeinflusst?

Das personalisierte Verhältniswahlrecht in Deutschland ist charakterisiert durch die Kombination von einfacher Mehrheitswahl (Erststimme) und Verhältniswahl (Zweitstimme) und bietet damit eine Möglichkeit die Stimmen strategisch

7 Wenn die Vermeidung der Stimmvergeudung das vorherrschende Motiv bei der Vergabe der Erststimme ist, verwenden Pappi und Herrmann (2006, S. 3) den Begriff „minimal strategisches Wählen“. 
zu splitten (Herrmann 2010, S. 669). ${ }^{8}$ Die Motive und Formen des strategischen Stimmensplittings können vielseitig sein (siehe z. B. Linhart 2007; Gschwend u. Pappi 2004; Pappi u. Thurner 2002; Schoen 1998). Wenn sich die ,Stimmensplitter' strategisch verhalten, d. h. Erststimme und Zweitstimme bewusst unterschiedlichen Parteien geben, weil sie z. B. eine bestimmte Koalition wünschen, dann dürfte es für das Splittingverhalten dieser Personen vollkommen unerheblich sein, ob der Direktkandidat einen Doktortitel trägt oder nicht.

Johann (2009, S. 418) bezweifelt, dass Stimmensplitter grundsätzlich „als die Prototypen des rationalen Wählers angesehen werden können“. Anknüpfend an das sozialpsychologische Wählermodell der Michigan School sieht er im „Persönlichkeitsbonus“ des Kandidaten ein alternatives Motiv für Stimmensplitting. Die Erststimme wird dem Kandidaten einer anderen Partei gegeben, weil dieser als attraktiver, sympathischer oder kompetenter wahrgenommen wird als der Kandidat der eigenen Partei (Johann 2009, S. 402). Wenn sich die Stimmensplitter vom ,Persönlichkeitsbonus' eines Kandidaten leiten lassen, d. h. responsiv gegenüber unpolitischen Kandidateneigenschaften sind, könnte der Doktortitel parteispezifische Wirkung entfalten. Im Hinblick auf eine abnehmende Parteibindung jüngerer Wähler (Eith 2006), einer steigenden Zahl an Wechselwählern (Weßels 2007) und zunehmender Kurzfristigkeit der Wahlentscheidung (Dalton u. Bürklin 2003) könnte der Persönlichkeitsbonus an Bedeutung gewonnen haben. Allerdings bietet auch diese Sichtweise keine abschließende Antwort auf die Frage, in welcher Partei der Doktortiteleffekt dominant wirken könnte. Vielmehr stellt sich die grundsätzliche Frage, ob parteispezifische Doktortitel-Effekte möglich sind. Letzteres würde zumindest aus der Perspektive des strategischen Stimmensplittings ausgeschlossen. Vor dem Hintergrund dieses schwachen theoretischen Fundaments, wollen wir daher die folgende allgemeine Hypothese prüfen:

\section{H4: Der positive Effekt des Doktortitels auf den Stimmenanteil eines Di- rektkandidaten variiert in Abhängigkeit von der Parteizugehörigkeit des Kandidaten.}

Frog-Pond-Effekt: Die Signalwirkung des Doktortitels könnte davon abhängen, ob die übrigen Kandidaten im Wahlkreis ebenfalls einen Doktortitel tragen. Dieser Effekt entspricht strukturell dem „Frog-Pond“-Effekt von Rosar, Klein und Beckers (2008, S. 76), die nachweisen konnten, dass physisch attraktive Kandidaten besser abschneiden, je niedriger die durchschnittliche Attraktivität aller Kandidaten im Wahlkreis ist. ${ }^{9}$ Diese Art der Wechselwirkung zwischen dem Individualmerkmal und der Verteilung des Merkmals auf der Gruppenebene ist ein in der Bildungsforschung bekanntes Phänomen (Davis 1966; Johanson 1993; Alicke

8 Siehe BVerfGE 95, 355. Im Juli 2008 stellte das Bundesverfassungsgericht fest (BVerfGE 3.7.2008, $2 \mathrm{BvC}$ 1/07), dass das Wahlrecht gegen den Grundsatz der gleichen Wahl verstößt und damit verfassungswidrig ist (vgl. Holzner 2010, S. 167). Die Wahl des 17. Deutschen Bundestages erfolge nach dem alten, verfassungswidrigen Wahlrecht.

9 Anstelle der Bezeichnung „Frog-Pond“ wird in der Literatur auch der Begriff „Big-Fish-LittlePond“-Effekt verwendet. Der Bezug zum Sprichwort „Lieber ein großer Fisch in einem kleinen Teich, als ein kleiner Fisch in einem großen" ist offenkundig. 
et al. 2010; Marsh 2005). In einer Klasse mit überwiegend leistungsschwachen Schülern neigen leistungsstarke Schüler zu einer Überbewertung ihrer eigenen Leistungsfähigkeit, während leistungsschwache Schüler in einer Klasse mit überwiegend leistungsstarken Klassenkameraden ihre eigenen Leistungen unterschätzen (Alicke et al. 2010, S. 174).

Im Kern bringt der Frog-Pond-Effekt die menschliche Neigung zum Ausdruck, in Vergleichssituationen relative anstelle von absoluten Maßstäben anzulegen. Absolut meint, dass $e x$ ante und unabhängig von den zu vergleichenden Objekten Kriterien der Beurteilung bestimmt werden. Relativ hingegen meint, dass die zu vergleichenden Objekte lediglich in eine Reihenfolge gebracht werden. Um absolute Vergleichsmaßstäbe anlegen zu können, sind umfassende allgemeine Kenntnisse über den Gegenstand notwendig. Da wir jedoch davon ausgehen können, dass die Beschaffung und Verarbeitung dieser Informationen Kosten verursacht, ist der Frog-Pond-Effekt selbst das Ergebnis einer Entscheidungsheuristik, die in Vergleichssituationen zum Einsatz kommt.

Der Frog-Pond-Effekt lässt sich auf die Wirkung des Doktortitels innerhalb eines Wahlkreises übertragen. Die Kandidaten werden von den Wählern nicht für sich alleine stehend betrachtet, sondern in Relation zu ihren Mitstreitern. Der Stimmenzugewinn des Kandidaten mit Doktortitel ist demnach größer, wenn es im gesamten Wahlkreis nur eine Person mit Doktortitel gibt. Der Doktortitel ist in diesem Fall ein herausragendes Merkmal des Kandidaten. Derselbe Kandidat würde jedoch weniger Stimmen für seinen Titel erhalten, wenn andere Kandidaten im Wahlkreis ebenfalls einen Doktortitel trügen. Die Signalwirkung des Titels wird also relativiert, wenn mehrere Kandidaten einen Doktortitel tragen.

\section{H5: Der positive Effekt des Doktortitels auf den Stimmenanteil nimmt ab, je mehr Kandidaten innerhalb des Wablkreises einen Doktortitel tragen.}

Es sei darauf hingewiesen, dass der postulierte positive Zusammenhang zwischen Doktortitel und Stimmenanteil in H5 im Vergleich zu H1 nicht etwa schwächer ist, sondern ein konzeptionell anderer Effekt. Die in H5 beschriebene Wirkung des Doktortitels ist gewissermaßen der ,Netto-Doktortiteleffekt ${ }^{\star}$, d. h., es handelt sich um den erwarteten positiven Nettoeffekt des Doktortitels, der geringer ausfallen sollte, wenn andere Kandidaten ebenfalls einen Doktortitel tragen. ${ }^{10}$

\subsection{Alternative Erklärungen und weitere Heuristiken}

Die Auswahl alternativer Erklärungen für die Stimmenabgabe stützt sich auf die Begründung des Bundestagspräsidenten. Demnach werden drei Erklärungen für den Wahlerfolg des Direktkandidaten unterschieden (Bundestag 2009, S. 2): die Parteizugehörigkeit, das öffentliche Wirken und das berufliche Wirken. Die Partei-

10 Auf Wahlkreisebene wurden weitere Kontexteffekte untersucht. Dazu zählen das Bildungsniveau, die Arbeitslosenquote sowie die Stimmenkonzentration, gemessen durch den Herfindahl-Index (Litz 2003, S. 117-118). Keine dieser Variablen hatte einen überzufälligen direkten oder konditionalen Effekt auf das Wahlergebnis. 
zugehörigkeit des Kandidaten ist sicherlich eines der stärksten Kriterien, das aus der Wählerperspektive für oder gegen die Stimmabgabe spricht. Diese Einschätzung deckt sich mit den Ausführungen von Rahn (1993) und anderen (z. B. Downs 1957; Steenbergen 2010), wonach Parteienlabel als Entscheidungsheuristik genutzt werden. Durch ein Parteienlabel werden vielschichtige und undurchsichtige Problematiken zu einem politischen Programm komprimiert, das der Wählerschaft dauerhaft Orientierung bieten soll. Die Vermutung, dass der Wahlerfolg vom öffentlichen Wirken des Direktkandidaten beeinflusst wird, steht ebenfalls in Einklang mit bisherigen Forschungsergebnissen zur Wirkungsweise von information shortcuts (Popkin 1993). Der Amtsinhaber verfügt tendenziell über eine bessere Ressourcenausstattung und ist im Wahlkreis in der Regel bekannter, z. B. durch Medienpräsenz und öffentliche Auftritte (z. B. Mackenrodt 2008, S. 75; Zittel u. Gschwendt 2007). In diesem Zusammenhang muss auch der Prominentenstatus eines Kandidaten genannt werden, den er oder sie durch Mitgliedschaft in der Bundesregierung oder den Fraktionsvorsitz im Bundestag erlangen kann (Mackenrodt 2008, S. 76; siehe auch Pappi u. Shikano 2001). Das berufliche Wirken wird als dritte Erklärung für den Wahlerfolg des Direktkandidaten angeführt (Bundestag 2009, S. 2). Die Befunde von McDermott (2005) für die USA liefern Hinweise, dass der berufliche Hintergrund des Kandidaten vom Wähler als Entscheidungsheuristik herangezogen wird. Die Wähler würden demnach die Politiknähe des Berufes des Kandidaten als Informationshilfe für die Beurteilung von politischen Fähigkeiten nutzen.

Weitere Entscheidungshilfen, die sich in der Literatur etabliert haben und daher ebenfalls in unserer Analyse berücksichtig werden, sind das Geschlecht und Alter des Kandidaten. Mit dem Geschlecht sind immer noch stereotype Eigenschaftszuschreibungen verbunden (McDermott 1997; Huddy u. Terkildsen 1993; Piliavin 1987; Rule 1987; Koch 2002). Männer werden mit Macht und Leistung assoziiert, Frauen hingegen mit Einfühlungsvermögen und sozialer Kompetenz. Hinsichtlich des Alters könnte vermutet werden, dass besonders junge Kandidaten als unerfahren eingestuft werden, während besonders alten Kandidaten ein Mangel an Robustheit unterstellt wird (Klein u. Rosar 2005, S. 280; Mackenrodt 2008, S. 76). ${ }^{11}$

Unser Modell zur Schätzung des Zusammenhangs zwischen Doktortitel und Wahlergebnis wird folgende alternative Erklärungen berücksichtigen: die Parteizugehörigkeit, den Zweitstimmenanteil der Partei des Kandidaten, Alter, Geschlecht und beruflichen Hintergrund, ob der Kandidat bereits zum Zeitpunkt der Wahl Mitglied im Bundestag war oder ein prominentes Amt bekleidete, ob der Kandidat ebenfalls einen Listenplatz hatte und ob der Kandidat einen Professortitel führt. Sollte der Doktortitel-Effekt neben diesen vom Bundestagspräsidenten angeführten und in der Literatur identifizierten Erklärungsfaktoren Bestand ha-

11 Weitere Faktoren die in der Literatur diskutiert werden sind u. a. die Position des Kandidaten auf dem amtlichen Wahlzettel (Faas u. Schoen 2006; Kelley u. McAllister 1984), die physische Attraktivität (Klein u. Rosar 2005), lokale Herkunft (Mackenrodt 2008) und das Wahlrecht (Brettschneider 2002, S. 59-89). 
ben, wäre dies ein Indiz dafür, dass der Doktortitel grundsätzlich geeignet ist, das Wahlergebnis zu beeinflussen.

\section{Operationalisierung und Methode}

\subsection{Daten}

Die empirische Analyse verwendet die Daten des Bundeswahlleiters zur Bundestagswahl 2009. Im Gegensatz zu vergleichbaren Studien (z. B. Mackenrodt 2008; Klein u. Rosar 2005) werden alle Direktkandidaten berücksichtigt, deren Partei auf der Wahlliste vertreten war. Von den insgesamt 2195 Kandidaten, die sich bei der Bundestagswahl 2009 für ein Direktmandat bewarben, waren 187 Kandidaten sogenannte Einzelbewerbungen ohne Landesliste oder gehörten einer Partei an, die nicht auf der Landesliste vertreten war. Weil für diese Kandidaten keine Informationen über den Zweitstimmenanteil ihrer Partei vorliegen, können sie nicht in der Analyse berücksichtigt werden. Eine Reduktion des Datensatzes auf Kandidaten von CDU/CSU, SPD und Die Linke erfolgt bewusst nicht. Dafür sprechen drei Gründe. Erstens, ex ante gibt es kein theoretisches Argument, das den Ausschluss einzelner Kandidaten rechtfertigen würde. Zweitens, handelt es sich bei dem Datensatz um eine (quasi) Vollerhebung, deren Vorzüge nicht durch weitere Fallselektion beeinträchtigt werden soll. Drittens, besteht ein analytischer Schwerpunkt des Beitrags darin, den Doktortitel-Effekt im Kontext der Wettbewerbssituation im Wahlkreis zu untersuchen (insbesondere H3 und H5). Durch das Beschneiden des Datensatzes gingen relevante Kontextinformationen verloren.

\subsection{Abhängige und unabhängige Variablen}

Die abhängige Variable misst den Erststimmenanteil eines Kandidaten in Prozentpunkten. Unabhängige Variable von besonderem Interesse ist der akademische Titel des Kandidaten. Hier unterscheiden wir zwischen einem Doktor- und einem Professortitel; beide werden mit Dummyvariablen erfasst $(1=\mathrm{ja}$, andernfalls 0$)$, wobei die Gruppe der Bewerber ohne Doktor- und Professortitel als Referenzkategorie dient. ${ }^{12}$ Um die doktorgradspezifische Wirkung des Doktortitels auf den Wahlerfolg zu untersuchen, unterscheiden wir den Doktorentitel in sechs Fachbereichen: Jura, Wirtschaftswissenschaften, Medizin, Sozialwissenschaften, Ingenieurswissenschaften und Naturwissenschaften. ${ }^{13}$ Die Gewinnchance eines Kandidaten wird aus dem tatsächlichen Wahlergebnis abgeleitet (Gschwend 2007,

12 Eine weitere Unterscheidung zwischen Ehrendoktor, Ehrenprofessur, Universitätsprofessur und Fachhochschulprofessur wird nicht vorgenommen.

13 Bei der Zuordnung der Doktortitel zu Fachbereichen wurde wie folgt vorgegangen: Eindeutige Fälle (z. B. Ärzte, Juristen) wurden auf Basis der Angaben im Originaldatensatz kodiert. Konnten wir aus den verfügbaren Angaben keine Kodierung vornehmen, so folgte eine Recherche in den Internetauftritten der Kandidaten bzw. ihrer Parteien, die in der Regel alle nötigen Informationen enthielten. In seltenen Fällen wurde auf private Webseiten zurückgegriffen. Doktortitel in den Bereichen Erziehungs-, Sprach-, Literatur-, Sport-, Kulturwissenschaften und Philosophie wurden den Sozialwissenschaften zugeordnet. Bei der Zuordnung des Doktorgrades wurden auch die Professoren berücksichtigt. 
S. 11). Um zu bestimmen, wie aussichtsreich die Bewerbung eines Kandidaten war, wird für jeden Kandidaten die Differenz zwischen seinem Ergebnis und dem Ergebnis des Gewinners des Mandats bestimmt. ${ }^{14}$ Im Falle des Mandatsgewinners erhält die Variable den Wert „eins“ und für alle anderen Bewerber den Wert „eins plus Stimmendifferenz zum Mandatsgewinner“. Die konditionale Wirkung der Gewinnchance und der Parteizughörigkeit des Kandidaten auf den DoktortitelEffekt wird durch multiplikative Interaktionsterme modelliert. Zur Überprüfung des Frog-Pond-Effekts werden zwei alternative Operationalisierungen vorgeschlagen. Zunächst wird eine wahlkreisspezifische Variable gebildet, welche die Anzahl aller Kandidaten mit Doktortitel im Wahlkreis summiert. Wenn für den direkten Einfluss des Doktortitels kontrolliert wird, sollte diese Variable gemäß dem FrogPond-Effekt einen negativen Einfluss auf den Stimmenanteil des Kandidaten haben. Alternativ wird der Einfluss des, gewichteten' Doktortitels auf den Wahlerfolg getestet. Diese Variable erhält weiterhin den Wert null, wenn der Kandidat keinen Doktortitel führt. Für Kandidaten, die einen Doktortitel führen, ergibt sich der Wert ihres Titels aus der Anzahl aller Kandidaten im Wahlkreis geteilt durch die Anzahl aller Kandidaten im Wahlkreis mit Doktortitel. ${ }^{15}$ Die Werte wurden anschließend standardisiert, sodass die Variable Doktortitel (gewichtet) im Wertebereich 0 bis 1 liegt.

$\mathrm{Zu}$ den politischen Kontrollvariablen gehören das Zweitstimmenergebnis der Partei, welcher der Direktkandidat angehört, sowie fünf Dummy-Variablen für die Parteizugehörigkeit des Kandidaten (CDU/CSU, SPD, FDP, Grüne, Die Linke). Alle anderen Parteien werden zur Gruppe „Sonstige Parteien“ zusammengefasst und dienen als Referenzkategorie. ${ }^{16}$ Als Grundlage zur Klassifikation des beruflichen Hintergrunds der Kandidaten dient die zweistellige Berufsklassifikation des Mikrozensus. Diese wird in eine vereinfachte Form der Berufsklassifikation nach Blossfeld (Schimpl-Neimanns 2003) überführt, sodass wir fünf Gruppen unterscheiden können: manuelle Berufe, Techniker/Ingenieure, Dienstleistungsberufe, (Semi-)Professionen und Berufe in Verwaltung und Management. Die Referenzkategorie beinhaltet Kandidaten in Agrarberufen, Nichterwerbstätige sowie Berufe, die nicht in Blossfelds Klassifikation berücksichtigt werden (Studenten, Rentner und Hausfrauen). Das öffentliche Wirken des Kandidaten wird mit drei Variablen erfasst: ob der Kandidat gleichzeitig als Listenkandidat antritt $(1=\mathrm{ja}$, andernfalls $0)$; ob der Kandidat derzeit Mitglied des Bundestages ist $(1=\mathrm{ja}$, andernfalls 0$)$

14 Die Operationalisierung der Gewinnchance basiert auf der Annahme, dass die Differenz zwischen den Erststimmenergebnissen am Wahltag die Erwartungshaltung eines durchschnittlichen Wählers systematisch widerspiegelt (vgl. Gschwend u. Pappi 2004, S. 172).

15 In einem Wahlkreis mit acht Kandidaten, von denen nur ein Kandidat einen Doktortitel trägt beträgt der Wert des Doktortitels 8 ( = 8/1) für den Kandidaten mit Doktortitel. Wenn im selben Wahlkreis zwei der acht Kandidaten einen Doktortitel tragen, reduziert sich der Wert des Doktortitels für beide Kandidaten mit Doktortitel auf $4(=8 / 2)$.

16 In der Gruppe „Sonstige Parteien“ sind enthalten: die Bayernpartei (BP), Bürgerrechtsbewegung Solidarität (BüSo), Die Violetten, Deutsche Kommunistische Partei (DKP), Die Tierschutzpartei, Die Familien-Partei Deutschlands (FAMILIE), Marxistisch-Leninistische Partei Deutschlands (MLPD), Nationaldemokratische Partei Deutschlands (NPD), Partei Bibeltreuer Christen (PBC), Piraten Partei, Republikaner (REP), Rentnerinnen und Rentner Partei (RRP), Volksabstimmung und die Ökologisch-Demokratische Partei (ödp). 
und ob der Kandidat einen Prominentenstatus genießt $(1=j$ a, andernfalls 0$)$. Als prominent gelten alle Kandidaten, die entweder einen Ministerposten in der Bundesregierung der vorherigen Legislaturperiode innehatten oder aber den Fraktionsvorsitz ihrer Partei besaßen. Ebenfalls als prominent gelten die Parteivorsitzenden in der Zeit von 2005 bis zur Wahl 2009. Weitere Kontrollvariablen sind das Alter des Kandidaten (logarithmiert) und das Geschlecht $(1=$ weiblich, $0=$ männlich). Die Definition und die statistischen Kennwerte der Variablen sind in Appendix Tabelle 1 und 2 zusammengefasst.

\subsection{Statistisches Modell}

Hinsichtlich der statistischen Modellierung des Zusammenhangs zwischen Doktortitel und Wahlergebnis müssen drei Aspekte berücksichtigt werden: das Skalenniveau der abhängigen Variable, die Tatsache, dass es sich bei dem Datensatz (quasi) um eine Vollerhebung handelt und Abhängigkeiten zwischen Beobachtungen auf Wahlkreisebene.

Der Wertebereich der abhängigen Variable Erststimmenanteil liegt zwischen 0 und 100 Prozentpunkten. Wird der Einfluss des Doktortitels auf die abhängige Variable mit OLS geschätzt, könnten die geschätzten Werte den Wertebereich 0 bis 100 über- oder unterschreiten. Für diesen Fall schlagen Papke und Wooldridge (1996) vor, ein quasi-likelihood Schätzverfahren anzuwenden. Die Überprüfung der geschätzten Werte (für Modell 3 in Tabelle 2) ergab, dass bei keinem Kandidaten der geschätzte prozentuale Stimmenanteil über 100 liegt. Zudem gibt es nur acht Kandidaten (0,4 \% des Samples) bei denen der geschätzte Stimmanteil unter 0 liegt. Da die Schätzergebnisse unverändert bleiben, wenn wir diese Kandidaten aus dem Sample entfernen, haben wir uns entschieden mit dem OLS-Schätzer weiterzuarbeiten. Um allerdings sicherzustellen, dass die Transformation der abhängigen Variable keine Verzerrung verursacht, werden alle Modelle ebenfalls mit der abhängigen Variable absolute Stimmenanzahl geschätzt. Die Befunde dieser Analyse sind in Appendix Tabelle 3 zusammengefasst.

Der zweite Punkt betrifft die Art des Datensatzes. Im Gegensatz zu den in der empirischen Wahlforschung gängigen Umfragedaten, handelt es sich bei den Daten des Bundeswahlleiters um eine Vollerhebung. Mit Bezug auf die Reduktion des Datensatzes um Kandidaten für die kein Zweitstimmenergebnis existiert $(\mathrm{N}=187)$ und die Auseinandersetzung um die Verwendung von Signifikanztests bei derartigen Erhebungen (Behnke 2005; Kittel 2009), folgen wir der Einschätzung von Broscheid und Gschwend (2005, 2003). Signifikanztests sind auch bei Vollerhebungen anwendbar, auch wenn sie nicht das einzige Mittel der Stochastizitätsanalyse darstellen müssen (Broscheid u. Gschwend 2005, S. O-24). Das Wahlergebnis eines Kandidaten kann von einer Vielzahl von Faktoren beeinflusst werden, die weder erfasst wurden oder erfassbar sind. Aus diesem Grund scheint uns die Berücksichtigung der stochastischen Komponente in Form von Standardfehlern legitim. Anstatt also lediglich auf die Beziehungen zwischen den Variablen hinzuweisen, sollte auch die Unsicherheit bestimmt werden, mit der diese Beziehungen die sozialen Wirklichkeiten widerspiegeln (Broscheid u. Gschwend 2005, S. O-24). Dabei gilt weiterhin der Grundsatz, dass die statistische Signifikanz nur 
eine notwendige, aber keine hinreichende Bedingung für die substanzielle Relevanz der gefundenen Beziehungen ist (Broscheid u. Gschwend 2005, S. O-24). Folglich werden wir bei der Interpretation der Koeffizienten den substanziellen Effekt und die Unsicherheit gleichermaßen berücksichtigen. Diese Vorgehensweise deckt sich mit der Studie von Mackenrodt (2008, S. 79), in der ebenfalls Signifikanzniveaus ausgewiesen werden, um „die Nicht-Zufälligkeit der Korrelation“ zu betonen.

Der dritte Aspekt betrifft die Spezifikation des OLS Modells. Die Wahl des Direktkandidaten findet in 299 Bundestagswahlkreisen statt. Die Anzahl der Kandidaten, die in einem Wahlkreis gegeneinander antraten, lag im Durchschnitt bei 7,5 (min. 4, max. 12). Die insgesamt 2008 Kandidaten in unserem Datensatz können nicht als unabhängige Beobachtungen gewertet werden. Wir haben zunächst Wahlkreis-Dummies in das Modell aufgenommen. Ein Wald-Test hat jedoch gezeigt, dass Wahlkreis-Dummies keinen gemeinsamen signifikanten Einfluss haben. Wir schätzen den Zusammenhang zwischen den unabhängigen Variablen und dem Stimmanteil der Direktkandidaten daher mit auf Wahlkreisebene geclusterten Standardfehlern (Kennedy 2003, S. 372-388).

\section{Empirische Analyse}

\subsection{Deskriptive Befunde}

Hinsichtlich der Promotionshäufigkeit besteht eine starke Abweichung zwischen den Direktkandidaten und der Gesamtbevölkerung. Während nur rund ein Prozent der Gesamtbevölkerung über einen Doktortitel verfügt (Statistisches Bundesamt 2008, S. 71), sind es bei den Bundestagskandidaten rund elf Prozent. Von den in den Bundestag gewählten Kandidaten haben sogar rund 21 Prozent einen Doktortitel. Bei der Bundestagswahl 2009 haben Direktmandatsgewinner mit Doktortitel durchschnittlich 42,02 Prozent aller im Wahlkreis abgegeben Stimmen erhalten, während Direktmandatsgewinner ohne Doktortitel durchschnittlich 41,36 Prozent erreicht haben. Die Differenz erscheint zunächst marginal. Beachtet man jedoch den durchschnittlichen Erststimmenanteil aller Kandidaten, erhalten die Kandidaten mit Doktortitel im Durchschnitt 9,45 Prozentpunkte mehr Stimmen als Kandidaten ohne Doktortitel. Dieser Befund deutet an, dass Direktkandidaten nicht nur mit höherer Wahrscheinlichkeit promoviert sind, sondern auch, dass der Doktortitel mit einem besseren Wahlergebnis in Verbindung gebracht werden kann. 


\section{Abbildung 1: Kandidaten und Gewinner mit Doktortitel nach Bundeswahlkreisen}
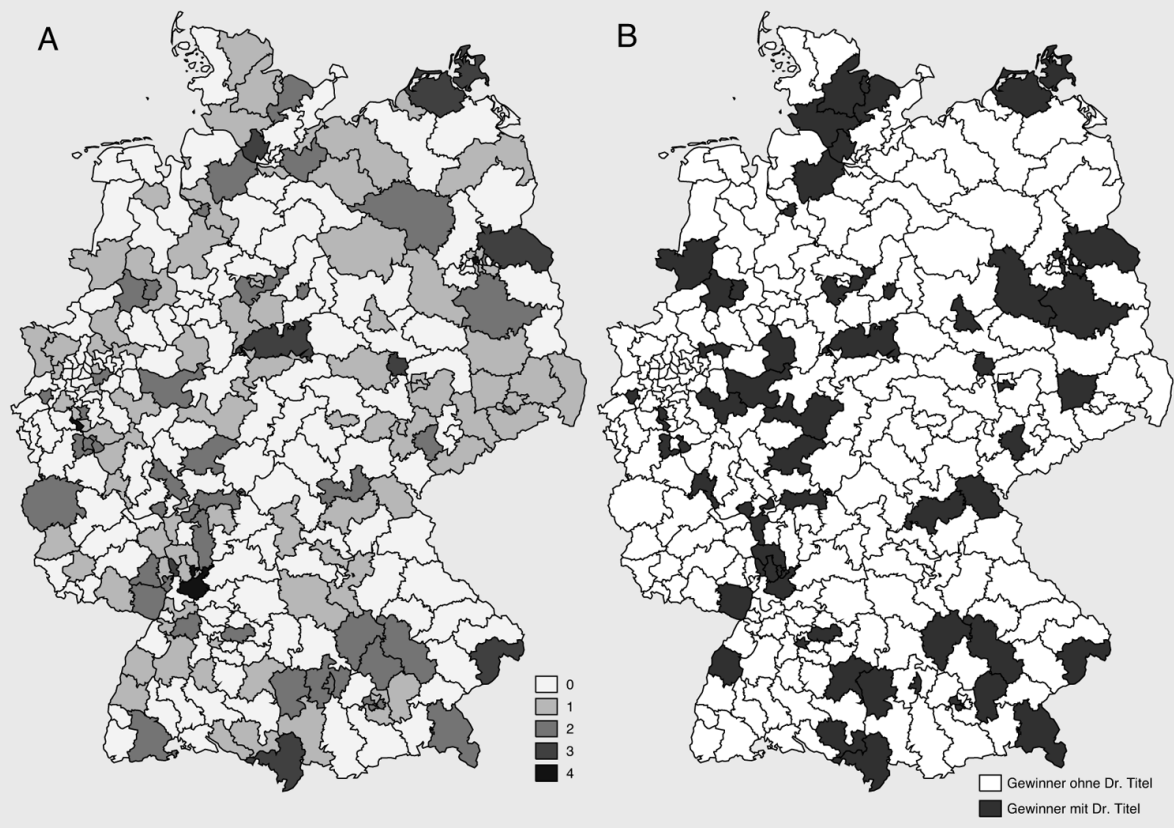

Hinweis: A) Anzahl der Kandidaten mit Doktortitel je Wahlkreis (o bis 4 Kandidaten mit Doktortitel), B) Gewinner des Direktmandats mit und ohne Doktortitel.

Abbildung 1 verschafft einen Überblick zur geographischen Verteilung der Kandidaten mit Doktortitel (Ausschnitt A) und der von Doktoren gewonnenen Wahlkreise (Ausschnitt B). Obwohl die politische Verteilung der Direktmandate einem geographischen Muster folgt, gilt dies für die räumliche Verteilung der Doktortitel nicht. Im Osten Deutschlands konkurrieren die Kandidaten der Partei Die Linke mit den Bewerbern von SPD und CDU, während in Bayern nahezu alle Direktmandate von der CSU gewonnen wurden. Eine räumliche Konzentration, die diesem Muster folgt, lässt sich weder für die Kandidaten (Abschnitt A) noch für die Mandatsgewinner (Abschnitt B) erkennen. Allerdings zeigt die Abbildung, dass in knapp der Hälfte aller Wahlkreise (48,2 \%) mindestens einer der Kandidaten einen Doktortitel führt. Von den insgesamt 144 Wahlkreisen mit mindestens einem Kandidaten mit Doktortitel treten in 36,8 Prozent gleich mehrere Doktoren gegeneinander an (max. 4 in einem Wahlkreis). Die Häufung von mehreren Kandidaten mit Doktortitel innerhalb eines Wahlkreises könnte als ein erstes Indiz für die wechselseitige Dynamik des Doktortitels innerhalb eines Wahlkreises gewertet werden.

Von den 211 Kandidaten mit Doktortitel haben 67 Personen einen Doktortitel im Bereich Sozialwissenschaften (davon 6 Professoren), 51 im Bereich Rechtswissenschaft (davon 1 Professor), 31 im Bereich Medizin, 29 im Bereich Wirtschaftwissenschaften (davon 1 Professor), 25 im Bereich Naturwissenschaften (davon 2 
Professoren) und 21 Kandidaten haben einen Doktortitel im Bereich der Ingenieurswissenschaften (davon 3 Professoren). Diese Verteilung bleibt im Kern erhalten, wenn wir uns die Fachbereichszughörigkeit der Gewinner eines Direktmandats anschauen (28 promovierte Juristen, 16 promovierte Sozialwissenschaftler, 11 promovierte Wirtschaftswissenschaftler, 5 promovierte Ingenieure, 4 promovierte Naturwissenschaftler und 3 promovierte Mediziner). Die Häufigkeitsverteilung weist darauf hin, dass die auf das Berufsprestige bezogene Erklärung doktorgradspezifischer Varianz geringe empirische Evidenz findet. Vielmehr zeichnet sich ab, dass Kandidaten mit einem Doktortitel in einem politiknahen Fachbereich bessere Chancen haben ein Direktmandat zu gewinnen.

Die meisten Direktkandidaten mit Doktortitel können die CDU/CSU $(20,40 \%)$ und die FDP $(14,38 \%)$ stellen, gefolgt von der SPD $(13,71 \%)$, den Grünen $(10,14 \%)$ und der Partei Die Linke $(7 \%)$. Bei den Direktmandatsträgern im Bundestag mit Doktortitel sieht es wiederum anders aus. Fast ein Drittel der Direktmandatsträger der Fraktion die Linke (31 \%) verfügt über einen Doktortitel. Bei der CDU verfügen 22,02 Prozent der Direktmandatsgewinner über einen Doktortitel und bei der SPD 17,19 Prozent. 


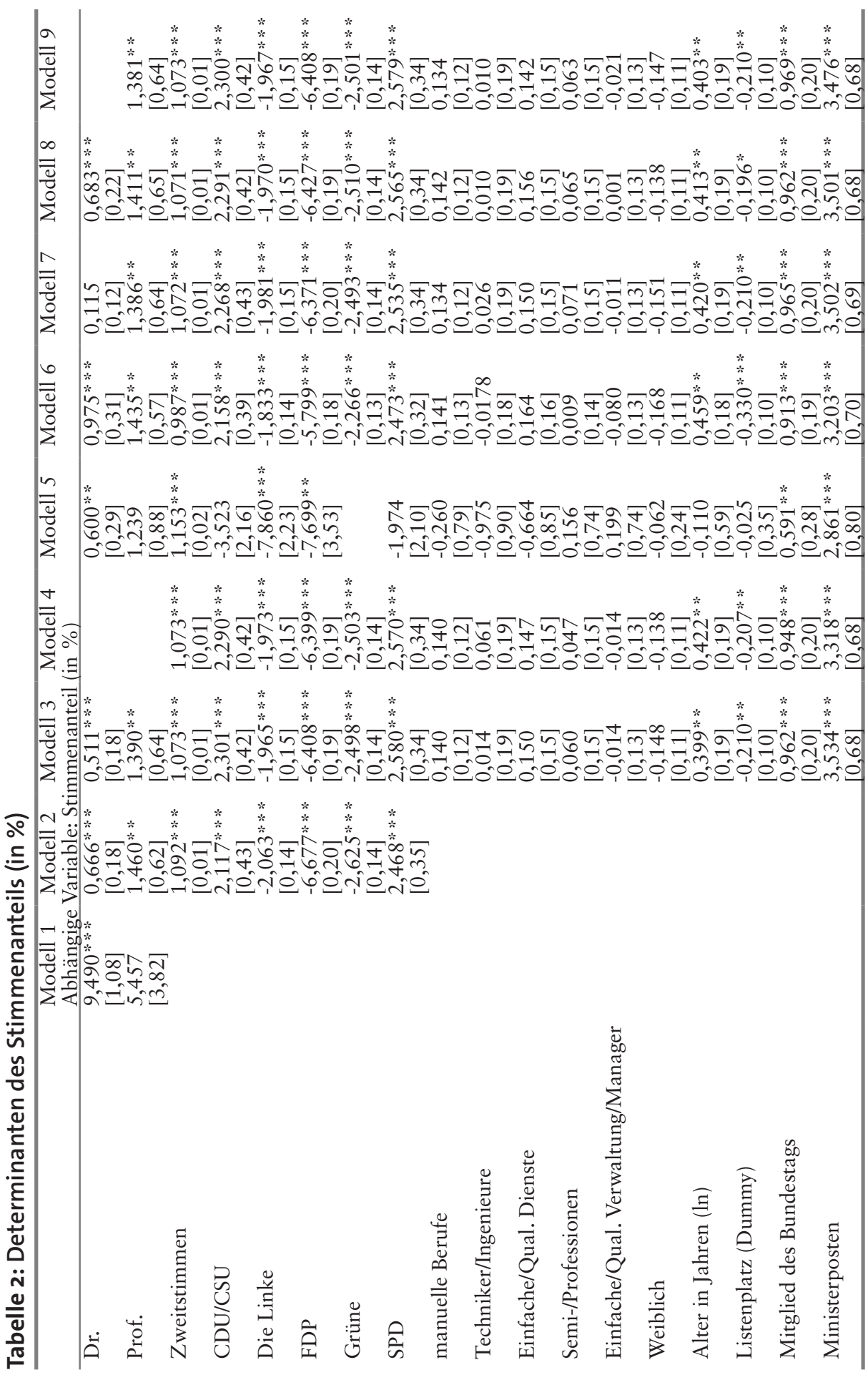




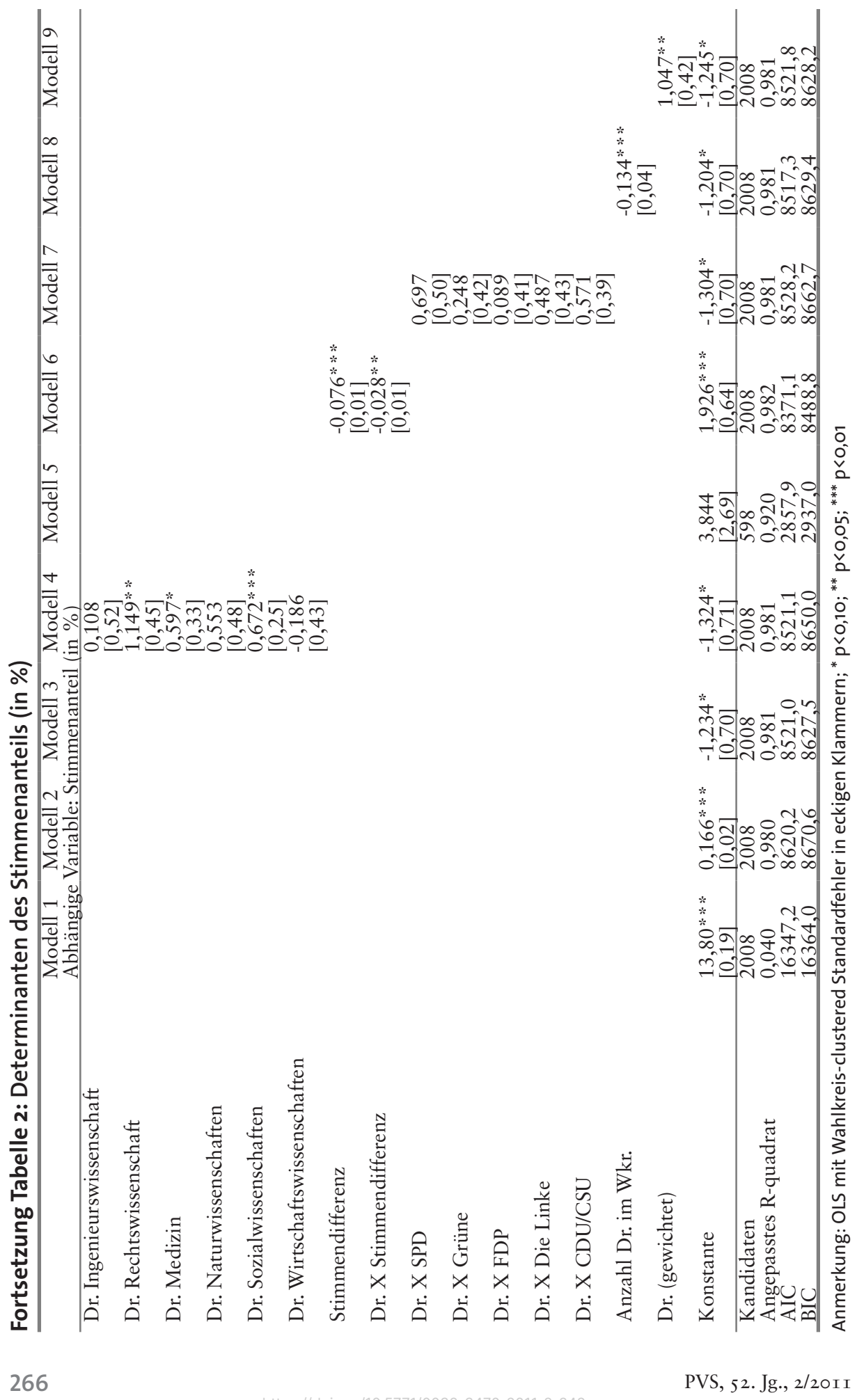




\subsection{Multivariate Analyse}

Die Ergebnisse der multivariaten Analyse sind in Tabelle 2 zusammengefasst. Die Modelle 1 bis 3 gelten der Überprüfung der Ausgangshypothese (H1). Dazu werden alternative Erklärungen, insbesondere jene aus der Begründung des Bundestagpräsidenten (2009), schrittweise in das Modell eingefügt. Die Modelle 4 bis 9 gelten der Überprüfung der Hypothesen H2-H5. ${ }^{17}$ Das Ausgangsmodell (Modell 1) beinhaltet lediglich zwei Dummy-Variablen für den Doktor- und Professortitel. Kandidaten mit Doktortitel gewinnen im Schnitt rund 9,5 Prozentpunkte mehr Erststimmen (entspricht 14.191 Stimmen) als Direktkandidaten ohne Doktorbzw. Professortitel. Der Regressionskoeffizient für einen Professortitel ist zwar ebenfalls positiv, aber nicht statistisch signifikant (Modell 1). Das ändert sich in Modell 2, welches der Argumentation des Bundespräsidenten folgend die Zweitstimmen und die Parteizugehörigkeit des Kandidaten berücksichtigt. Der Effekt des Doktortitels schrumpft auf einen Stimmengewinn von rund 0,7 Prozentpunkten (entspricht 2279 Stimmen). Der Effekt des Professortitels auf den Wahlerfolg ist nunmehr größer als der Effekt des Doktortitels und ist ebenfalls signifikant. Hier sollte allerdings beachtet werden, dass von den insgesamt 2008 Kandidaten nur 14 Personen einen Professortitel tragen. ${ }^{18}$

Der Effekt der Parteizugehörigkeit ist nicht nur überzufällig, sondern in substanzieller Hinsicht auch einflussreicher als der Doktortitel-Effekt. Größe und Vorzeichen der Koeffizienten bestätigen, dass der Großteil der Direktmandate von CDU/CSU-Kandidaten (2,12 Prozentpunkte, p-Wert = 0,00) bzw. von SPD-Kandidaten (2,47 Prozentpunkte, p-Wert = 0,00) gewonnen wird. Für Kandidaten der anderen Parteien ist der Zusammenhang zwischen Parteizugehörigkeit und Stimmenanteil negativ. Das Zweitstimmenergebnis der Partei des Direktkandidaten ist der wichtigste Prädiktor für den Wahlerfolg des Direktkandidaten. Erhöht sich der Zweitstimmenanteil der Partei des Direktkandidaten um eine Standardabweichung (entspricht 11,94 Prozentpunkten) erhöht sich der Stimmenanteil des Direktkandidaten bereits um 13,04 Prozentpunkte (Modell 2). Die Pearson-Korrelation zwischen Erst- und Zweistimmenanteil liegt bei bemerkenswerten 0,97 $(\mathrm{p}$-Wert $=0,000)$. Es ist im Wesentlichen dieser Variable geschuldet, dass das angepasste R-Quadrat von 0,04 in Modell 1 auf 0,98 in Modell 2 hinaufschnellt. Die Inklusion weiterer erklärender Faktoren hat kaum Einfluss auf das angepasste R-Quadrat.

Entgegen der Annahme des Bundestagspräsidenten zeigt Modell 3, dass der berufliche Hintergrund weder einen substanziellen noch einen überzufälligen Einfluss auf den Wahlerfolg hat. Mit steigendem Alter (logarithmiert) erhöht sich auch der Stimmenanteil. Bei einer Alterspanne von 71 Lebensjahren ist der Maximaleffekt des Alters (plus 0,64 Prozentpunkte) in substanzieller Hinsicht vergleichbar mit dem Doktortitel-Effekt. Das Geschlecht hat keinen signifikanten Einfluss auf den Stimmenanteil. Kandidaten, die bereits im Bundestag vertreten

17 Die Ergebnisse der Analyse mit der absoluten Stimmenzahl als abhängige Variable sind in Appendix Tabelle 3 zusammengefasst.

18 Ein Kandidat führt einen Ehrenprofessor ohne Promotion. 
sind, erzielen durchschnittlich 0,96 Prozentpunkte mehr (1225,6 Stimmen absolut) als Neubewerber. Somit fällt auch der Bonus, den der gegenwärtige Mandatsträgers besitzt, stärker aus als der Doktortitel-Effekt. Die gleichzeitige Besetzung eines Listenplatzes wird mit einer leichten Reduzierung des Stimmenanteils in Verbindung gebracht (-0,21 Prozentpunkte oder -335 Stimmen). ${ }^{19}$ Kandidaten, die bereits durch die Übernahme eines Ministeramtes oder Fraktionsvorsitzes in der Öffentlichkeit standen, holen rund 3,5 Prozentpunkte mehr Stimmen (4764,6 Stimmen absolut). Dies deckt sich mit den bisherigen Erkenntnissen, dass Amtsinhaber und bekannte Kandidaten über einen ,Promibonus' verfügen. ${ }^{20}$

Insgesamt unterstützen die Modelle 1 bis 3 die Ausgangshypothese H1. Allerdings haben die in der Begründung des Bundestagspräsidenten angeführten alternativen Erklärungen einen substanziellen Einfluss auf das Wahlergebnis des Kandidaten. So schmilzt der Doktortitel-Effekt von 9,5 Prozentpunkten (entspricht 14.191,8 Stimmen) im Basismodell (Modell 1) auf grade noch 0,51 Prozentpunkte (entspricht 739,9 Stimmen) im vollständigen Modell (Modell 3). In substanzieller Hinsicht fällt der Doktortitel-Effekt im Vergleich zu politischen Determinanten (insbesondere Zweitstimmenanteil, Parteizugehörigkeit, Ministerposten) eher gering aus. Dennoch ist der Effekt deutlich signifikant und robust gegenüber Veränderungen der Modellspezifikation. Bei der Bundestagswahl 2009 gab es in insgesamt acht Wahlkreisen ein Ergebnis bei dem die Stimmendifferenz zwischen Erst- und Zweitplatziertem unter 0,51 Prozentpunkten lag, d. h., in diesen Wahlkreisen hätte der Doktortitel potenziell wahlentscheidend sein können.

Modell 4 überprüft, ob der Zusammenhang zwischen Doktortitel und Wahlerfolg mit dem Doktorgrad variiert. Doktoren der Rechtswissenschaften gewinnen im Schnitt zusätzliche 1,15 Prozentpunkte (entspricht 1782 Stimmen), Doktoren der Sozialwissenschaften 0,67 Prozentpunkte (entspricht 990 Stimmen) und Mediziner 0,60 Prozentpunkte (entspricht 1046 Stimmen) gegenüber Kandidaten ohne Doktortitel. Nur in diesen drei Fällen ist der doktorgradspezifische Effekt überzufällig. Entgegen der aus dem allgemeinen Berufsprestige abgeleiteten Vermutung ist der Doktortitel-Effekt sowohl für Juristen als auch für Sozialwissenschaftler etwas stärker als für Mediziner. Das Ergebnis deckt sich mit den deskriptiven Befunden, wonach die Mehrzahl der Gewinner eines Direktmandates mit Doktortitel in einem politiknahen Fachbereich promoviert sind. Daraus könnte abgeleitet werden, dass nicht das mit dem Berufsfeld bzw. dem Fachbereich assoziierte Prestige, sondern die mit dem Fachbereich assoziierte politische Expertise die Chance auf ein Direktmandat erhöht. In diesem Fall muss allerdings die Frage gestellt werden, warum keine Effekte von einer Promotion in Wirtschaftswissenschaften ausgehen, zumal die Wirtschaftswissenschaften ebenfalls als politiknahe Profession einzustufen sind (z. B. mit Blick auf die Wirtschafts-, Haushalts- oder

19 Die Verwendung des Listenrangplatzes anstelle des Listenplatz-Dummies zeigt, dass die besonders hohen Listenrangplätze für den negativen Zusammenhang verantwortlich sind.

20 Ein Adelstitel könnte ebenfalls den Prominentenstatus eines Kandidaten steigern. Die statistische Überprüfung des Zusammenhangs zwischen Adelstitel $(\mathrm{N}=14)$ und Wahlerfolg erwies sich zwar als positiv aber nicht überzufällig. 
Steuerpolitik). ${ }^{21}$ Welcher Wirkmechanismus tatsächlich für die doktorgradspezifische Varianz verantwortlich ist, kann auf Basis der Befunde nicht eindeutig geklärt werden. Insgesamt sollte der Befund aber vor dem Hintergrund der zum Teil sehr geringen Fallzahlen (insb. Medizin und Naturwissenschaft) nicht überbewertet werden.

Mit Modell 5 und 6 wird untersucht, wie sich die Gewinnchancen des Kandidaten auf den Doktortitel-Effekt auswirken. Es wird angenommen, dass der Doktortitel-Effekt irrelevant wird, je schlechter der Kandidat im Vergleich zum Gewinner des Mandats abschneidet, d. h. je ,chancenloser' der Kandidat, desto unwichtiger wird der Doktortitel. Die Überprüfung dieses Argumentes stellt uns vor eine methodische Herausforderung, da sich die Stimmendifferenz zum Gewinner des Mandats unmittelbar aus dem Wahlergebnis des Kandidaten ergibt. Zittel und Gschwend (2007, S. 302-303) operationalisieren die Wettbewerbsintensität über eine dichotome Variable, die erfasst, ob im vorherigen Wahlkampf die Wahl für die Partei des Kandidaten mit mehr als zehn Prozentpunkten Unterschied zum Sieger verloren ging oder nicht. Da wir jedoch davon ausgehen müssen, dass sich die Konstellationen im Wahlkreis seit 2005 verändert haben können, z. B. durch neue Kandidaten im Wahlkreis (Fluktuation), die allgemeine bundespolitische Lage usw., ist diese Operationalisierung für unsere Fragestellung eher ungeeignet. Zunächst wird der Doktortitel-Effekt auf reduzierten Samples bestehend aus Erstund Zweitplatzierten (Modell 5), Zweit- und Drittplatzierten sowie Dritt- und Viertplatzierten ermittelt. Die Fallzahl reduziert sich jeweils auf $2 \times 299=598$ Kandidaten. Erst im zweiten Schritt wird die Interaktion zwischen Dr. $x$ Stimmendifferenz zum Erstplatzierten modelliert (Modell 6). Das Interaktionsmodell hat den Vorteil, dass wir überprüfen können, ob sich das Wahlergebnis für Doktoren und Nicht-Doktoren mit unterschiedlichen Gewinnchancen signifikant unterscheidet. Der Nachteil dieses Verfahrens ist die Endogenität der Stimmendifferenzvariable. Mit der Reduzierung des Samples auf die Erst- und Zweitplatzierten bzw. die Zweit- und Drittplatzierten etc. wird das Endogenitätsproblem behoben, allerdings zu dem Preis, dass wir nun nicht mehr in der Lage sind zu bestimmen, ob sich das Wahlergebnis für Doktoren und Nicht-Doktoren mit unterschiedlichen Gewinnchancen signifikant unterscheidet.

Modell 5 in Tabelle 2 basiert auf dem reduzierten Sample bestehend aus Erstund Zweitplatzierten. Der Effekt des Doktortitels ist nun mit 0,60 Prozentpunkten (entspricht 818 Stimmen) um rund 0,10 Prozentpunkte größer als der Doktortitel-Effekt im Basismodell (Modell 3). Wird dieselbe Modellspezifikation auf dem Sample bestehend aus Zweit- und Drittplatzierten und dem Sample der Dritt- und

21 Alternativ haben wir den direkten Einfluss der Magnitude-Prestigeskala (MPS) von Wegener (1992) auf den Stimmenerfolg des Direktkandidaten überprüft. Der MPS-Wert eines Kandidaten hatte keinen Einfluss auf dessen Wahlerfolg. Zudem wurde der konditionale Effekt des MPSWertes auf die Stärke des Doktortitel-Effektes überprüft. Auch in diesem Fall konnten keine überzufälligen Zusammenhänge festgestellt werden. 
Viertplatzierten geschätzt, ist der Doktortitel-Effekt nicht länger signifikant und sinkt von $0,42\left(\mathrm{p}-\right.$ Wert=0,215) auf 0,24 Prozentpunkte $\left(\mathrm{p}\right.$-Wert=0,304). ${ }^{22}$

\section{Abbildung 2: Konditionale Wirkung der Stimmendifferenz zum Mandatsgewinner auf den Doktortitel-Effekt}

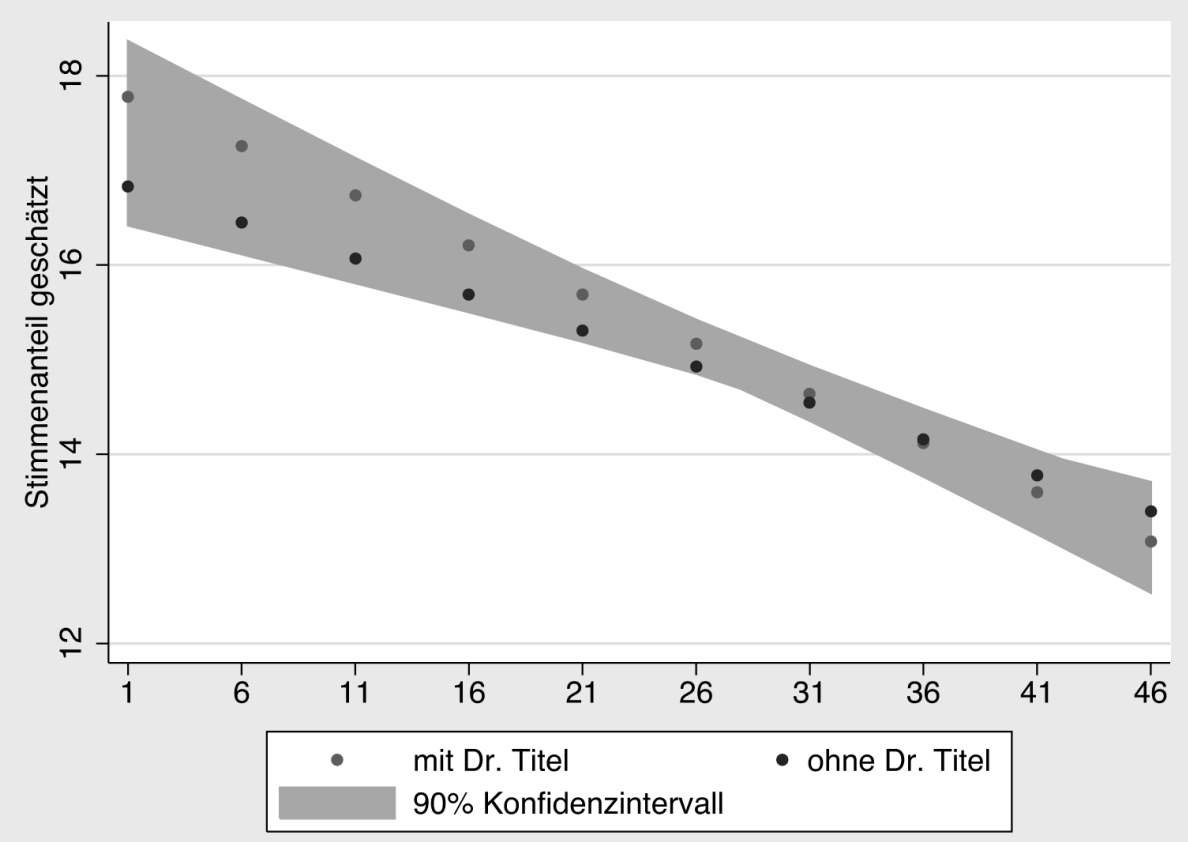

Anmerkung: Basierend auf Modell 6.

Der konditionale Einfluss der Gewinnchance, gemessen als Stimmendifferenz zum Mandatsgewinner, wird mit Modell 6 untersucht. Abbildung 2 zeigt den geschätzten Erststimmenanteil eines Kandidaten in Abhängigkeit von seiner Stimmendifferenz zum Direktmandatsgewinner. Wie zu erwarten war, sinkt der geschätzte Erststimmenanteil mit steigender Stimmendifferenz zum Mandatsgewinner. Bemerkenswert hingegen sind die Unterschiede in der Steigung der Funktion für Kandidaten mit bzw. ohne Doktortitel. Bis zu einer Stimmendifferenz von 31 Prozentpunkten nimmt der Doktortitel-Effekt kontinuierlich ab. Während die Gewinner des Direktmandates durch den Doktortitel zusätzliche Stimmen im Umfang von 0,95 Prozentpunkten erhalten, schrumpft der Stimmenzuwachs auf 0,10 Prozentpunkte, wenn die Stimmendifferenz zum Erstplatzierten 31 Prozentpunkte beträgt. Nach diesem Punkt kreuzen sich die beiden Funktionen und der Doktortitel-Effekt wird negativ. In nur 21 Wahlkreisen hatte der Zweitplatzierte eine

22 Die Subsample-Regressionen für Zweit- und Drittplatzierte und Dritt- und Viertplatzierte sind nicht in Tabelle 2 ausgewiesen. Der vollständige Datensatz und die Analysesyntax stehen für Replikaktionszwecke zur Verfügung. 
Stimmendifferenz zum Mandatsgewinner von mehr als 31 Prozentpunkten. Mit Blick auf die Parteizugehörigkeit sind es vorrangig die Kandidaten von der FDP, den Grünen, Die Linke und Bewerber anderer kleiner Parteien, die eine höhere Stimmendifferenz aufweisen. Dieses Muster steht im Einklang mit der Vermutung, dass die Wähler gemäß der Logik der einfachen Mehrheitswahl ihre Erststimmen nicht vergeuden wollen, sondern einsetzen, um einen der beiden aussichtsreichsten Kandidaten zu wählen (Herrmann 2010, S. 667). Abbildung 2 legt den Schluss nahe, dass der Doktortitel nur für die beiden aussichtsreichsten Kandidaten mit einem positiven Effekt auf den Erststimmenanteil in Verbindung gebracht werden kann. In allen anderen Fällen hat der Doktortitel keinen substanziellen und positiven Einfluss auf das Erststimmenergebnis.

Dieser Befund muss allerdings vor dem Hintergrund des sich zum Teil deutlich überschneidenden 90\%-Konfidenzintervalls vorsichtig interpretiert werden. Bis zu einer Stimmendifferenz von 21 Prozentpunkten ist die Überschneidung des oberen Konfidenzintervalls für Kandidaten ohne Doktortitel und dem unteren Konfidenzintervall für Kandidaten mit Doktortitel vergleichsweise gering (unter 0,05 Prozentpunkte). Erst danach liegen die Konfidenzintervalle deutlich übereinander, sodass nicht davon ausgegangen werden kann, dass sich das geschätzte Erststimmenergebnis für die beiden Gruppen in irgendeiner Weise überzufällig unterscheidet. Trotz dieser Einschränkung unterstützen die gemeinsamen Befunde aus Modell 5 (Subsample-Regression) und Modell 6 (Interaktionsmodell) die Hypothese H3, wonach der Doktortitel-Effekt schwächer werden sollte, wenn die Gewinnchance des Kandidaten abnimmt.

Modell 7 prüft die konditionale Wirkung der Parteizugehörigkeit des Bewerbers auf den Doktortitel-Effekt. Keiner der insgesamt fünf Interaktionsterme ist statistisch signifikant. Dieser Befund bleibt unverändert, wenn die Interaktionsterme separat in das Modell aufgenommen werden. Ein Wald-Test für die gemeinsame Signifikanz der Interaktionsterme ergibt für Dr. X CDU und Dr. X SPD einen F-Wert von $1,88(\mathrm{p}=0,15)$, für die anderen drei Interaktionsterme wird ein FWert von $0,50(\mathrm{p}=0,68)$ festgestellt. Dieses Muster wird bestätigt, wenn anstelle des prozentualen Wahlergebnisses die absolute Stimmenzahl als abhängige Variable verwendet wird (s. Appendix Tabelle 3). Die Ergebnisse liefern folglich keine empirische Evidenz für eine parteispezifische Varianz in der Wirkung des Doktortitels (H4). In substantieller Hinsicht ist der Interaktionskoeffizient für die SPDBewerber etwas größer als für CDU/CSU-Kandidaten, gefolgt von Kandidaten der Partei Die Linke, Grüne und FDP. Dass die Kandidaten der beiden ,großen' Parteien tendenziell eher von einem Doktortitel profitieren, kann vor dem Hintergrund des Ticket-Splitting interpretiert werden. Der empirische Regelfall ist, dass die Wahl eines Direktmandats zwischen dem CDU/CSU- und dem SPD-Kandidaten entschieden wird. Bei der Bundestagswahl 2009 gab es nur 62 Wahlkreise, in denen Kandidaten der FDP, der Grünen oder Linken einen der ersten beiden Rangplätze besetzen konnten. Um herauszufinden, ob CDU/CSU-Direktkandidaten in substanzieller Hinsicht stärker von einem Doktortitel profitieren als SPDDirektkandidaten, haben wir den Stimmenanteil für beide Gruppen von Kandidaten jeweils mit und ohne Doktortitel geschätzt. Die Differenz zwischen dem geschätzten Stimmenanteil für einen CDU/CSU-Kandidaten mit Doktortitel und 
einem CDU/CSU-Kandidaten ohne Doktortitel beträgt 0,68 Prozentpunkte (entspricht 938 Stimmen). Für SPD-Kandidaten ist die Differenz mit 0,81 Prozentpunkte etwas größer (entspricht 1367 Stimmen). In beiden Fällen ist die Differenz nicht signifikant, sodass $\mathrm{H} 4$ insgesamt abgelehnt werden muss.

Die Wirkung des Frog-Pond-Effekts wird mit Modell 8 und 9 überprüft. Zunächst wird die Anzahl der Kandidaten mit Doktortitel auf Wahlkreisebene in Modell 8 aufgenommen. Unter Berücksichtigung des Doktortitel-Effektes reduziert ein weiterer Kandidat mit Doktortitel das Wahlergebnis eines Bewerbers um 0,13 Prozentpunkte (entspricht 200,3 Stimmen absolut). Für einen Kandidaten ohne Doktortitel heißt das, er würde in einem Wahlkreis, in dem kein Kandidat einen Doktortitel führt, einen Stimmenanteil von 14,85 Prozentpunkten erwarten. Derselbe Kandidat würde 0,54 Prozentpunkte Stimmen weniger erhalten, wenn 4 Kandidaten in seinem Wahlkreis einen Doktortitel tragen. Noch bei 99\%-Konfidenzintervallen handelt es sich um eine signifikante Differenz. In Stimmen ausgedrückt beträgt der maximale Verlust 802 Stimmen, ein Stimmenverlust, der allein daraus resultiert, dass andere Kandidaten im Wahlkreis einen Doktortitel tragen.

\section{Abbildung 3: Der Frog-Pond-Effekt}

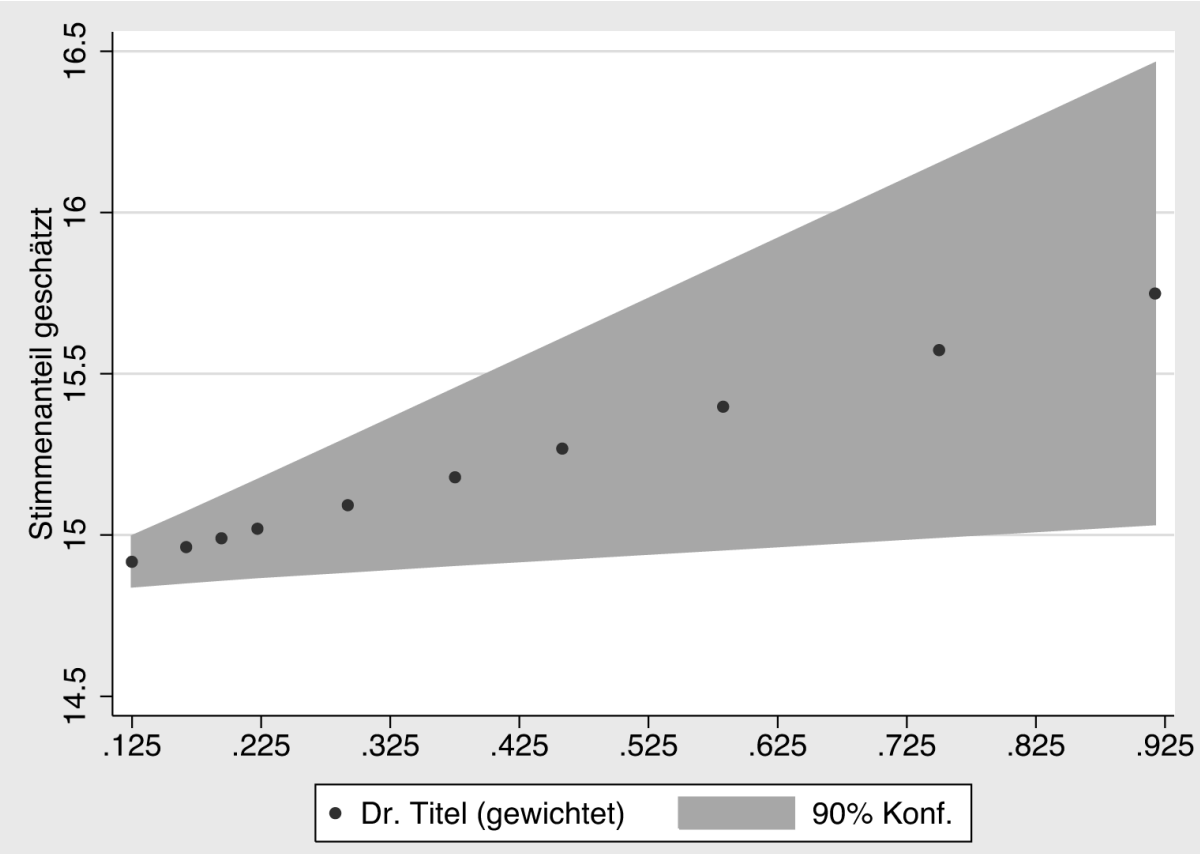

Anmerkung: Basierend auf Modell 9.

Anstelle der Dummy-Variable verwendet Modell 9 ein gewichtetes Maß für den Doktortitel. Die Variable behält den Wert „null“, wenn der Kandidat keinen Doktortitel trägt. Wenn der Kandidat einen Doktortitel trägt, wird die Anzahl aller Kandidaten im Wahlkreis durch die Zahl der Kandidaten mit Doktortitel ge- 
teilt, d. h. höhere Werte zeigen an, dass der Kandidat mit Doktortitel durch dieses Merkmal vergleichsweise ,besonders' ist, während niedrige Werte (aber nicht null) anzeigen, dass der Kandidat zwar einen Doktortitel trägt aber mit diesem Merkmal nicht hervorsticht. Die Variable ist auf einen Wertebereich von 0 bis 1 standardisiert, sodass der Maximaleffekt einem Stimmenzuwachs von 1,05 Prozentpunkten (bzw. 1485 Stimmen) entspricht. Der Einfluss der Variable auf den geschätzten Stimmenanteil ist in Abbildung 3 dargestellt. Die Darstellung bestätigt, dass mit zunehmender ,Besonderheit ${ }^{`}$ des Doktortitels im Wahlkreis der positive Effekt des Doktortitels auf den Wahlerfolg zunimmt. Allerdings wird ebenfalls deutlich, dass sich die 90\%-Konfidenzintervalle der Schätzung drastisch ausweiten, sofern die ,Besonderheit ${ }^{`}$ des Doktortitels hohe Werte annimmt. Dieser Befund sollte vor dem Hintergrund der zum Teil sehr geringen Fallzahl bewertet werden. In der Summe bestätigen die Befunde aus Modell 8 und 9 jedoch den Eindruck, dass der Zusammenhang zwischen Doktortitel und Wahlerfolg dem in H5 beschriebenen Frog-Pond-Effekt folgt. ${ }^{23}$

23 Ein dritter Vorschlag zur Prüfung des Frog-Pond-Effekts besteht darin zwei neue Dummy-Variablen zu verwenden: Die Variable Einziger Dr. im Wablkreis erhält den Wert 1, wenn es im Wahlkreis des Kandidaten keinen weiteren Kandidaten gibt, der über einen Doktortitel verfügt. Die Variable Nicht Einziger Dr. im Wablkreis erhält den Wert 1, wenn der Kandidat über einen Doktortitel verfügt und es im Wahlkreis mindestens einen weiteren Kandidaten mit Doktortitel gibt. Als Referenzgruppe dienen Kandidaten ohne Doktor- bzw. Professortitel. Im Vergleich zur Referenzgruppe steigt der Stimmanteil für Kandidaten, die als einzige einen Doktortitel im Wahlkreis tragen, um 0,69 Prozentpunkte (entspricht 1005 Stimmen), während der Effekt für Kandidaten in einem Wahlkreis mit mehreren Doktoren mit 0,38 Prozentpunkten (entspricht 545 Stimmen) schwächer ausfällt. In beiden Fällen ist der Effekt statistisch signifikant und liefert weitere empirische Evidenz für H5. 
Abbildung 4: Geschätztes Wahlergebnis für den Wahlkreis Steinfurt III
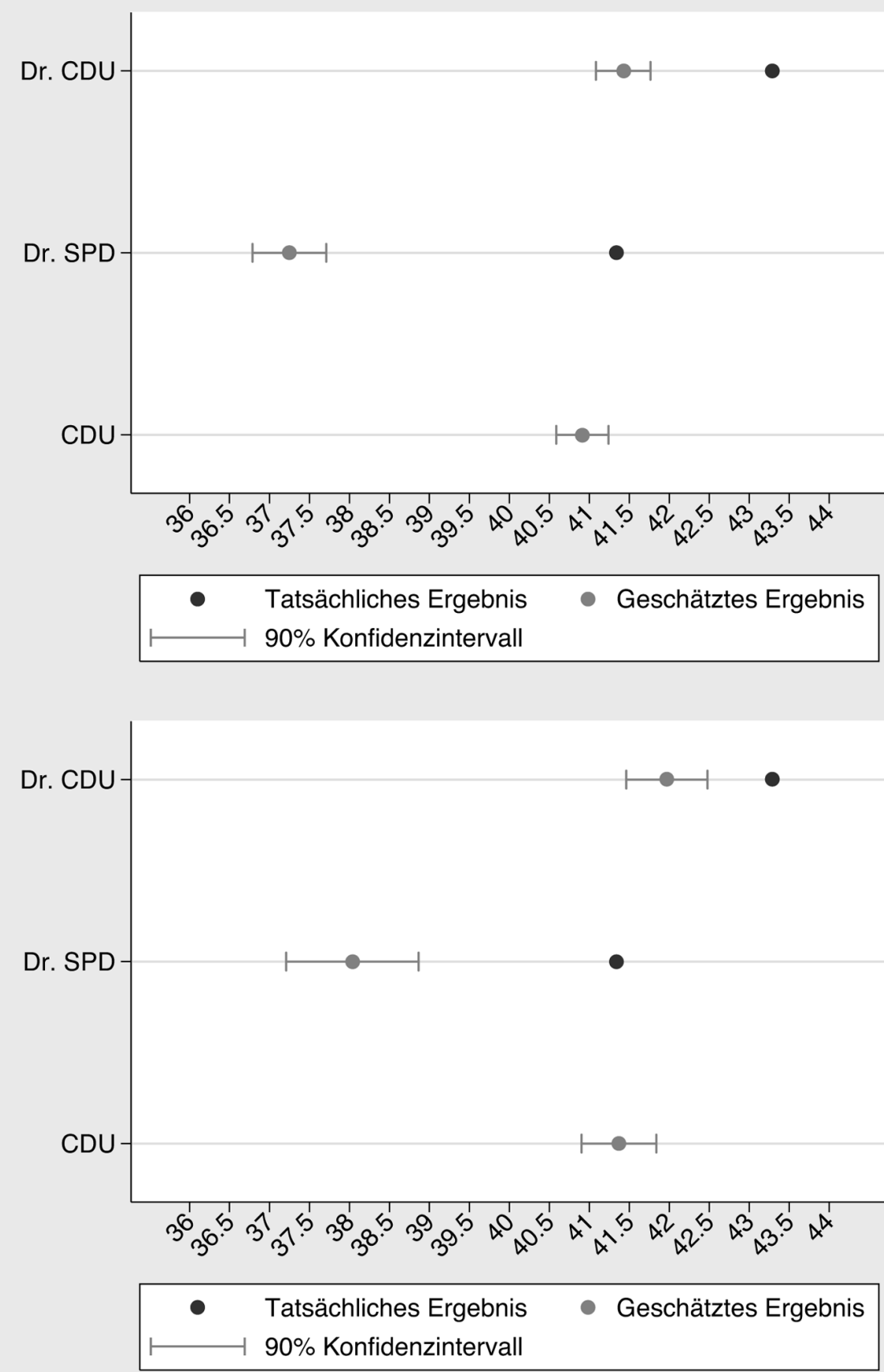

Anmerkung: Basierend auf Modell 3 und 5 . 


\subsection{Prognose für den Wahlkreis Steinfurt III}

Zum Abschluss der empirischen Analyse soll geklärt werden, ob der ,falsche“ Doktortitel im Wahlkreis Steinfurt III wahlentscheidend war. Welches Ergebnis hätte sich in dem Wahlkreis ergeben, wenn der CDU-Kandidat keinen Doktortitel vorgegeben hätte? Um diese Frage zu beantworten, werden die Modelle 3 und 5 aufgegriffen und die Stimmanteile für den SPD-Kandidaten mit Doktortitel und den CDU-Kandidaten mit und ohne Doktortitel geschätzt. Modell 3 wird verwendet, weil dieses Modell mit einem angepassten R-Quadrat von 0,98 die beste Anpassung aufweist. Modell 5 wird aufgegriffen, weil die Analyse gezeigt hat, dass die Relevanz des Doktortitel-Effektes von der Gewinnchance des Kandidaten abhängt.

Abbildung 4 zeigt das tatsächliche Wahlergebnis für den CDU- und SPD-Kandidaten sowie das geschätzte Ergebnis mit dem dazugehörigen 90\%-Konfidenzintervall. Die letzte Zeile zeigt jeweils das geschätzte Ergebnis des CDU-Kandidaten mit dem dazugehörigen 90\%-Konfidenzintervall, wenn dieser keinen Doktortitel getragen hätte. Um zu beurteilen, ob der Doktortitel wahlentscheidend war, werden die geschätzten Werte verglichen. Beide Schätzungen zeigen, dass der CDUKandidat durch den Doktortitel einen höheren Stimmenanteil erreicht hat. In Modell 3 beträgt sein geschätzter Stimmenanteil 41,42 Prozent mit und 40,91 Prozent ohne Doktortitel. In Modell 5 beträgt sein Stimmenanteil 41,97 Prozent mit und 41,37 Prozent ohne Doktortitel. Das unrechtmäßige Führen des Doktortitels kann demnach mit einen Stimmenzuwachs von 0,51 bis 0,60 Prozentpunkten in Verbindung gebracht werden.

Um zu beurteilen, ob diese Differenz wahlentscheidend war, muss das geschätzte Ergebnis des SPD-Kandidaten berücksichtigt werden. Dessen Stimmenanteil liegt in Modell 3 bei 37,25 Prozent und in Modell 5 bei 38,04 Prozent. Die 90\%-Konfidenzintervalle der Punktschätzung des Stimmenanteils des SPD-Kandidaten und des CDU-Kandidaten ohne Doktortitel zeigen in beiden Fällen keine Überschneidung. Unabhängig davon, ob der CDU-Kandidat einen Doktortitel getragen hätte, wäre der SPD-Kandidat mit sehr hoher Wahrscheinlichkeit unterlegen gewesen. In diesem Punkt bestätigen die Ergebnisse der Regressionsanalyse die Einschätzung des Bundestagspräsidenten (2009, S. 2). Der ,falsche‘ Doktortitel war mit sehr hoher Wahrscheinlichkeit nicht wahlentscheidend. Die Genauigkeit des geschätzten Wahlergebnisses sollte allerdings vor dem Hintergrund der Modellgüte kritisch reflektiert werden. Zwar ist die Modellanpassung in Modell 3 und 5 ausgesprochen hoch, dennoch verbleiben 1,9 Prozent (Modell 3) bzw. 8 Prozent (Modell 5) unerklärte Varianz in der abhängigen Variable.

\section{Zusammenfassung und Schlussfolgerungen}

Welchen Einfluss hat der Doktortitel auf den Wahlerfolg eines Direktkandidaten? Diese Frage bildete den Ausgangspunkt der empirischen Analyse. Die Ergebnisse lassen sich in drei Punkten zusammenfassen: Erstens, ein Doktortitel hatte bei der Bundestagswahl 2009 einen positiven und statistisch signifikanten Einfluss auf den Wahlerfolg der Direktkandidaten. Unter Berücksichtigung alternativer politi- 
scher und unpolitischer Kandidateneigenschaften erhöht ein Doktortitel den Erststimmenanteil im Durchschnitt um 0,51 Prozentpunkte. Die Befunde legen den Schluss nahe, dass der Doktortitel grundsätzlich geeignet ist den Wahlerfolg zu beeinflussen (H1). Zweitens, die Art und Stärke des Zusammenhangs zwischen Doktortitel und Wahlerfolg werden von Kontextfaktoren beeinflusst. Mit steigender Stimmendifferenz zwischen Kandidat und Mandatsgewinner scheint der Doktortitel-Effekt schwächer zu werden (H3), d. h. ein chancenloser Bewerber kann sein Wahlergebnis nicht durch einen Doktortitel substanziell verbessern. Für den Frog-Pond-Effekt sind die empirischen Hinweise noch deutlicher. Je niedriger die durchschnittliche Anzahl von Kandidaten mit Doktortitel auf Wahlkreisebene, desto besser schneiden Kandidaten mit Doktortitel ab (H5). Weitere Kontexthypothesen, die sich auf den Doktorgrad (H2) und die Parteizugehörigkeit des Kandidaten (H4) beziehen, finden keine empirische Unterstützung. Drittens, die Prognose des Wahlergebnisses für den Wahlkreis Steinfurt III ergibt, dass der ,falsche“ Doktortitel trotz des knappen Wahlergebnisses mit sehr hoher Wahrscheinlichkeit nicht wahlentscheidend war.

Bevor die potenziellen Implikationen der statistischen Befunde diskutiert werden, müssen die methodischen Einschränkungen und Grenzen der empirischen Analyse benannt werden. Mit einem Querschnittsdatensatz, wie er in diesem Beitrag verwendet wird, können keine Aussagen zur Kausalität der Effekte getroffen werden. Wenn wir einen statistisch signifikanten Zusammenhang zwischen dem Doktortitel und dem Erststimmenanteil eines Kandidaten beobachten, kann daraus nicht gefolgert werden, dass der Doktortitel ursächlich für das Wahlergebnis ist. Es wäre z. B. ebenso denkbar, dass politisch talentierte Kandidaten frühzeitig erkennen, dass ein Jurastudium mit anschließender Promotion die besten Voraussetzungen schafft, um in Deutschland eine politische Karriere zu machen. Auf Basis der hier verwendeten tatsächlichen Wahlergebnisdaten kann zudem nur überprüft werden, ob die aus Überlegungen zum individuellen Wahlverhalten abgeleiteten Hypothesen mit den im Aggregat beobachtbaren Wahlergebnissen in Einklang stehen. Ob die Annahmen über individuelles Wahlverhalten tatsächlich für das Wahlergebnis ursächlich sind, d. h. aus welchen Gründen Wähler einen Doktortitel als Entscheidungshilfe heranziehen und wie sie das Signal Doktortitel kognitiv verarbeiten, lässt sich am ehesten mit Hilfe von Umfragedaten oder Wahlexperimenten untersuchen. In welchem Umfang der Doktortitel-Effekt auf eine selektive Berichterstattung zurückzuführen ist, könnte im Rahmen einer Medienanalyse näher bestimmt werden.

Wie verhalten sich die empirischen Befunde hinsichtlich der Entscheidung des Bundestagspräsidenten, die nachträgliche Prüfung eines Wahlmangels durch den Wahlprüfungsausschuss abzulehnen? Grundsätzlich muss der Einspruch auf Wahlprüfung binnen einer Frist von zwei Monaten nach dem Wahltag beim Bundestag eingehen. Das der CDU-Kandidat im Wahlkreis Steinfurt III den gekauften Doktortitel nicht hätte auf dem amtlichen Wahlzettel angeben dürfen, wurde erst nach Ablauf dieser Frist bekannt. In diesem Fall sieht das Wahlprüfungsgesetz vor, dass der Bundestagspräsident nachträglich Einspruch einlegen kann, wenn ihm Umstände bekannt werden, die einen Wahlmangel begründen könnten ( $\$ 2 \mathrm{Abs.} 4$ Satz 2 WPrüfG). Ob die empirischen Befunde einen nachträglichen Einspruch auf 
Wahlprüfung rechtfertigen, hängt davon ab, welche Anforderungen an die Begründetheit des $\mathbb{2}$ Abs. 4 Satz 2 WPrüfG gestellt werden. Wenn die Perspektive eingenommen wird, dass die Formulierung „grundsätzlich geeignet wahlentscheidend zu sein" ein Kriterium darstellt, ergibt sich aus dem empirischen Befund, dass der Einspruch auf nachträgliche Prüfung eines Wahlmangels begründet war. Wenn die Formulierung hingegen in eine notwendige („grundsätzlich geeignet“) und eine hinreichende (,wahlentscheidend“) Bedingung zerlegt wird, war der Einspruch unbegründet, weil der ,falsche` Doktortitel zwar grundsätzlich geeignet, aber nicht wahlentscheidend war. Welche Anforderung an die Begründetheit des $\mathbb{2}$ Abs. 4 Satz 2 WPrüfG die, richtige' ist, ist eine originär juristische Frage.

Eine problematische Dynamik könnte der Doktortitel-Effekt dadurch entfalten, dass die Wirkung des Titels von sich strategisch verhaltenden Kandidaten antizipiert wird. Unter Unsicherheit und Informationsmangel können die Kandidaten erwarten, dass Wähler den Doktortitel direkt oder vermittelt über die Medien als Entscheidungshilfe nutzen (Popkin 1993; Maurer u. Schoen 2010). Es kann also vermutet werden, dass die Kandidaten ein Eigeninteresse haben den Doktortitel zwecks Stimmenmaximierung gezielt einzusetzen. Unter diesen Umständen ist zu erwarten, dass es auch in Zukunft Direktkandidaten geben wird, die zu Unrecht einen Doktortitel auf dem amtlichen Wahlzettel angeben. Vor diesem Hintergrund erscheint es sinnvoll, die Hürden für die Begründetheit des $\mathbb{} 2$ Abs. 4 Satz 2 WPrüfG niedrig anzusetzen, d. h. der nachträgliche Einspruch wäre begründet, wenn das Mittel "grundsätzlich geeignet“ ist. Die Abwägung wie wahrscheinlich es ist, dass ein ,falscher' Doktortitel tatsächlich wahlentscheidend war, würde dem Wahlprüfungsausschuss überantwortet. Aufgrund seiner Zusammensetzung ist dieses Gremium grundsätzlich am besten geeignet eine Abwägung zwischen dem Anspruch auf Behebung des Wahlmangels und dem Bestandsschutz des einmal gewählten Parlamentes vorzunehmen. Im konkreten Fall hätte dem nachträglichen Einspruch gem. $\mathbb{S} 2$ Abs. 4 Satz 2 WPrüfG stattgeben werden sollen. Im Anschluss hätte der Wahlprüfungsausschuss nach empirischer Prüfung zum selben Ergebnis gelangen müssen wie der Bundestagspräsident, denn auch ohne, falschen' Doktortitel hätte der CDU-Kandidat mit großer Wahrscheinlichkeit gewonnen, was sehr stark dafür spricht, dem Bestandschutz des Bundestags Vorrang vor der Behebung des Wahlmangels durch Neuwahl einzuräumen.

\section{Literatur}

Alicke, Mark D., Ethan Zell, und Dorian L. Bloom. 2010. Mere Categorization and the Frog Pond Effect. Psychological Science 21:174-177.

Allensbacher Institut. 2008. Allensbacher Berufsprestige-Skala 2008. http://www.ifd-allensbach.de/news/prd_0802.html. Zugegriffen: 7.10.2010.

Behnke, Joachim. 2005. Lassen sich Signifikanztests auf Vollerhebungen anwenden? Einige essayistische Anmerkungen. Politische Vierteljahresschrift 46:1-15.

Berliner Morgenpost. 2008. Der Hochstapler von Landau. 10. Juni 2008. http://www.morgenpost.de/printarchiv/politik/article215952/Der_Hochstapler_von_Landau.html. Zugegriffen: 11.03.2011. 
Brettschneider, Frank. 2002. Spitzenkandidaten und Wablerfolg. Personalisierung, Kompetenz, Parteien. Ein internationaler Vergleich. Wiesbaden: Westdeutscher Verlag.

Broscheid, Andreas, und Thomas Gschwend. 2003. Augäpfel, Murmeltiere und Bayes: Zur Auswertung stochastischer Daten aus Vollerhebungen. MPIfG Working Paper 03/7. Köln: Max-Planck-Institut für Gesellschaftsforschung.

Broscheid, Andreas, und Thomas Gschwend. 2005. Zur statistischen Analyse von Vollerhebungen. Politische Vierteljahresschrift 46:O-16-O-26.

Bryman, Alan. 2008. Social Research Methods. Oxford: Oxford University Press.

Bundestag. 2009. Schreiben Dr. Norbert Lammert an Kathrin Vogeler zur Wahlprüfung im Fall Dieter Jasper (03.03.2010). http://www.muensterschezeitung.de/storage/med/ muenst/pdf/5724_Ablehnung_Lammert.pdf. Zugegriffen: 7.10.2010.

Bundeswahlleiter. 2009. Wahl zum 17. Deutschen Bundestag am 27. September 2009. http://www.bundeswahlleiter.de/de/bundestagswahlen/BTW_BUND_09/.Zugegriffen: 7.10.2010.

Dalton, Russell J., und Wilhelm Bürklin. 2003. Wähler als Wandervogel: Dealignment and the German Voter. German Politics and Society 21:57-75.

Davis, James A. 1966. The Campus as a Frog Pond: An Application of the Theory of Relative Deprivation to Career Decisions of College Men. American Journal of Sociology 72:17-31.

Downs, Anthony. 1957. An Economic Theory of Democracy. New York: Addison Wesley.

Eith, Ulrich. 2006. Parteibindungen bei jungen und älteren Erwachsenen in Westdeutschland. In Jugend und Politik: „Voll normal!“. Der Beitrag der politischen Soziologie zur Jugendforschung, Hrsg. Edeltraud Roller, Frank Brettschneider und Jan W. van Deth, 361-377. Wiesbaden: VS Verlag für Sozialwissenschaften.

Faas, Thorsten, und Harald Schoen. 2006. The Importance of Being First: Effects of Candidates List Positions in the 2003 Bavarian State Election. Electoral Studies 25:91-102.

Faas, Thorsten, Kai Arzheimer, und Sigrid Roßteutscher (Hrsg.). 2010. Information Wahrnehmung - Emotion. Wiesbaden: VS Verlag für Sozialwissenschaften.

Frankfurter Allgemeine Zeitung. 2011. Guttenberg tritt zurück. Frankfurter Allgemeine Zeitung 02.03.2011:1.

Gschwend, Thomas. 2007. Ticket-Splitting and Strategic Voting under Mixed Electoral Rules. Evidence from Germany. European Journal of Political Research 46:1-23.

Gschwend, Thomas, und Franz U. Pappi. 2004. Stimmensplitting und Koalitionswahl. In Die Bundestagswahl 2002, Hrsg. Frank Brettschneider, Jan van Deth und Edeltraud Roller, 167-183. Wiesbaden: VS Verlag für Sozialwissenschaften.

Herrmann, Michael. 2010. Wenn wenige den Ausschlag geben. Strategisches Erststimmenwählen bei den deutschen Bundestagswahlen 1994-2009. Politische Vierteljahresschrift 51:665-689.

Hoffmeyer-Zlotnik, Jürgen H. P., und Alfons Geis. 2003. Berufsklassifikation und Messung des beruflichen Status/Prestige. ZUMA Nachrichten 52:125-138.

Holzner, Thomas. 2010. Die Verfassungswidrigkeit des Bundeswahlrechts. Problemstellung und Lösungsansätze. Zeitschrift für Gesetzgebung 25:167-192.

Huddy, Leonie, und Nayda Terkildsen. 1993. Gender Stereotypes and the Perception of Male and Female Candidates. American Journal of Political Science 37:119-147.

Johann, David. 2009. Charakteristika und Motive der Stimmensplitter bei der Bundestagswahl 2005. In Wähler in Deutschland: sozialer und politischer Wandel, Gender und 
Wablverhalten, Hrsg. Steffen Kühnel, 399-423. Wiesbaden: VS Verlag für Sozialwissenschaften.

Johanson, George A. 1993. Absolute and Relative Grading Standards. Beitrag präsentiert auf dem Annual Meeting of the Amercian Educational Research Association, 1216.4.1993, Atlanta.

Kaack, Heino. 1981. Die personelle Struktur des 9. Deutschen Bundestages - ein Beitrag zur Abgeordnetensoziologie. Zeitschrift für Parlamentsfragen 12:165-203.

Kaack, Heino. 1988. Die soziale Zusammensetzung des Deutschen Bundestages. In USKongress und Deutscher Bundestag. Bestandesaufnahmen im Vergleich, Hrsg. Uwe Thaysen, Roger Davidson und Richard G. Livingston, 128-151. Opladen: Westdeutscher Verlag.

Kaase, Max. 1994. Is There Personalization in Politics? Candidates and Voting Behavior in Germany. International Political Science Review: 211-230.

Kelley, Jonathan, und Ian McAllister. 1984. Ballot Paper Cues and the Vote in Australia and Britain: Alphabetic Voting, Sex, and Title. Public Opinion Quarterly 48:452-466.

Kennedy, Peter. 2003. A Guide to Econometrics. Cambridge: MIT Press.

Kittel, Bernhard. 2009. Statistische Erfordernisse und Inferenzschlüsse in makroquantitativ vergleichenden Forschungsdesigns. In Methoden der vergleichenden Politik- und Sozialwissenschaft: neue Entwicklungen und Anwendungen, Hrsg. Susanne Pickel, Detlef Jahn, Hans-Joachim Lauth, Gert Pickel, 277-295. Wiesbaden: VS Verlag für Sozialwissenschaften.

Klein, Markus, und Ulrich Rosar. 2005. Physische Attraktivität und Wahlerfolg. Eine empirische Analyse am Beispiel der Wahlkreiskandidaten bei der Bundestagswahl 2002. Politische Vierteljahresschrift 46:263-287.

Kleining, Gerhard. 1975. Soziale Mobilität in der Bundesrepublik Deutschland II: Statusoder Prestige-Mobilität. Kölner Zeitschrift für Soziologie und Sozialpsychologie 27:273-292.

Koch, Jeffrey W. 2002. Gender Stereotypes and Citizens' Impressions of House Candidates' Ideological Orientations. American Journal of Political Science 46:453-462.

Lass, Jürgen. 1995. Vorstellungsbilder über Kanzlerkandidaten. Zur Diskussion um die Personalisierung von Politik. Wiesbaden: Deutscher Universitätsverlag.

Linhart, Eric. 2007. Rationales Wählen als Reaktion auf Koalitionssignale am Beispiel der Bundestagswahl 2005. Politische Vierteljahresschrift 48:461-484.

Litz, Hans Peter. 2003. Statistische Methoden in den Wirtschafts- und Sozialwissenschaften. München: Oldenbourg Verlag.

Mackenrodt, Christian. 2008. Wie wichtig ist die Person? Zur Bedeutung von Persönlichkeitsfaktoren von Wahlkreisbewerbern bei Bundestagswahlen. Zeitschrift für Parlamentsfragen 1/2008:69-83

Marsh, Herbert W. 2005. Big-Fish-Little-Pond Effect on Academic Self-Concept. Zeitschrift für Pädagogische Psychologie 19:119-127.

Maurer, Marcus, und Harald Schoen. 2010. Der mediale Attraktivitätsbonus. Wie die physische Attraktivität von Wahlkreiskandidaten die Medienberichterstattung in Wahlkämpfen beeinflusst. Kölner Zeitschrift für Soziologie und Sozialpsychologie 62:277295. 
McDermott, Monika L. 1997. Voting Cues in Low-Information Elections: Candidate Gender as a Social Information Variable in Contemporary United States Elections. American Journal of Political Science 41:270-283.

McDermott, Monika L. 2005. Candidate Occupations and Voter Information Shortcuts. Journal of Politics 67:201-219.

Merkur. 2010. Falscher Doktor: Moosburger Stadtrat muss zablen. 29.04.2010. http:// www.merkur-online.de/lokales/nachrichten/falscher-doktor-moosburger-stadtrat-musszahlen-739766.html. Zugegriffen: 7.10.2010.

Papke, Leslie E., und Jeffrey M. Wooldridge. 1996. Econometric Methods for Fractional Response Variables with an Application to 401 (K) Plan Participation Rates. Journal of Applied Econometrics 11:619-632.

Pappi, Franz Urban, und Michael Herrmann. 2006. Strategisches Erststimmenwählen bei deutschen Bundestagswahlen. MZES Working Paper Nr. 92. Mannheim: Mannheimer Zentrum für Europäische Sozialforschung

Pappi, Franz Urban, und Paul W. Thurner. 2002. Electoral Behaviour in a Two-Vote System: Incentives for Ticket Splitting in German Bundestag Elections. European Journal of Political Research 41:207-232.

Pappi, Franz Urban, und Susumu Shikano. 2001. Personalisierung der Politik in Mehrparteiensystemen am Beispiel deutscher Bundestagswahlen seit 1980. Politische Vierteljabresschrift 42:355-387.

Piliavin, Jane Allyn. 1987. Age, Race, and Sex Similarity to Candidates and Voting Preference. Journal of Applied Social Psychology 17:351-368.

Popkin, Samuel. 1993. Information Shortcuts and the Reasoning Voter. In Information, Participation and Choice: An Economic Theory of Democracy in Perspective, Hrsg. Bernard Grofman, 17-35. Ann Arbor: Michigan University Press.

Rahn, Wendy M. 1993. The Role of Partisan Stereotypes in Information Processing about Political Candidates. American Journal of Political Science 37:472-496.

Riddel, Peter. 1993. Honest Opportunism. The Rise of Career Politician. London: Hamish Hamilton.

Rosar, Ulrich. 2009. Fabulous Front-Runners. Eine Analyse zur Bedeutung der physischen Attraktivität von Spitzenkandidaten für den Wahlerfolg ihrer Parteien und zu den Möglichkeiten der Gegensteuerung durch das Wahlrecht. Politische Vierteljahresschrift 50:754-773.

Rosar, Ulrich, Markus Klein, und Tilo Beckers. 2008. The Frog Pond Beauty Contest: Physical Attractiveness and Electoral Success of the Constituency Candidates at the North Rhine-Westphalia State Election of 2005. European Journal of Politcal Research 47:64-79.

Rosenthal, Allan. 1981. Legislative Life. New York: Harper \& Row.

Rule, Wilma. 1987. Electoral Systems, Contextual Factors and Women's Opportunity for Election to Parliament in Twenty-Three Democracies. Western Political Quarterly 40:477-498.

Sattar, Majiid. 2009. Und das bin ich! Guido Westerwelle - eine politische Biografie. München: Olzog Verlag.

Schimpl-Neimanns, Bernhard. 2003. Mikrodaten-Tools: Umsetzung der Berufsklassifikation von Blossfeld auf die Mikrozensen 1973-1998. ZUMA-Methodenbericht 2003/10. 
Schoen, Harald. 1998. Stimmensplitting bei Bundestagswahlen: eine Form taktischer Wahlentscheidung? Zeitschrift für Parlamentsfragen 29:223-244.

Spence, Michael. 1973. Job Market Signaling. Quarterly Journal of Economics 87:355374.

Spence, Michael. 2002. Signaling in Retrospect and the Informational Structure of Markets. American Economic Review 92:434-459.

Spiegel. 2009. CDU-Politiker soll falscher Doktor sein. 23.07.2009. http://www.spiegel.de/ unispiegel/jobundberuf/0,1518,637812,00.html. Zugegriffen: 7.10.2010.

Squire, Peverill. 1992. Legislative Professionalization and Membership Diversity in State Legislatures. Legislative Studies Quarterly 17:69-79.

Statistisches Bundesamt. 2008. Datenreport 2008. Wiesbaden: Statistisches Bundesamt.

Steenbergen, Marco R. 2010. The New Political Psychology of Voting. In Information Wahrnehmung - Emotion, Hrsg. Thorsten Faas, Kai Arzheimer und Sigrid Roßteutscher, 13-31. Wiesbaden: VS Verlag für Sozialwissenschaften.

Süddeutsche Zeitung. 2010. Der falsche Doktor von der CDU. 17.2.2010. http://www. sueddeutsche.de/politik/affaere-jasper-der-falsche-doktor-von-der-cdu-1.65396. Zugegriffen: 21.3.2011.

Wattenberg, Martin P. 1991. The Rise of Candidate-Centered Politics: Presidential Elections of the 1980s. Cambridge: Harvard University Press.

Wegener, Bernd. 1992. Concepts and Measurement of Prestige. Annual Review of Sociology 18:253-280.

Weßels, Bernhard. 2007. Re-Mobilisierung, "Floating” oder Abwanderung? Wechselwähler 2002 und 2005 im Vergleich. In Die Bundestagswahl 2005: Analysen des Wablkampfes und der Wahlergebnisse, Hrsg. Frank Brettschneider, Oskar Niedermayer und Bernhard Weßels, 395-419. Wiesbaden: VS Verlag für Sozialwissenschaften.

Zittel, Thomas, und Thomas Gschwend. 2007. Individualisierte Wahlkämpfe im Wahlkreis. Eine Analyse am Beispiel des Bundestagswahlkampfes von 2005. Politische Vierteljahresschrift 48:293-321.

\section{Autorenangaben}

Jun.-Prof. Dr. Markus Tepe,

Carl von Ossietzky Universität Oldenburg, Zentrum für Methoden der Sozialwissenschaften, Ammerländer Heerstraße 114-118, 26129 Oldenburg,

markus.tepe@uni-oldenburg.de

[Korrespondenzautor]

Dipl. Soz.-Wiss. Sebastian Schneider,

Carl von Ossietzky Universität Oldenburg, Zentrum für Methoden der Sozialwissenschaften, Ammerländer Heerstraße 114-118, 26129 Oldenburg, sebastian.schneider@uni-oldenburg.de 


\section{Appendix Tabelle 1: Definition der Variablen}

\begin{tabular}{|c|c|}
\hline Variable & Definition \\
\hline Stimmenanteil (in \%) & Anteil der erzielten Erststimmen im Wahlkreis in Prozent \\
\hline Akademischer Titel & $\begin{array}{l}\text { Dr. }(1=\text { ja, andernfalls } 0) \text {, Prof. }(1=j a \text {, andernfalls } 0) \text { Referenz } \\
=\text { kein Doktor-, oder Professortitel. }\end{array}$ \\
\hline Dr. Titel nach Fachbereich & $\begin{array}{l}\text { Fachbereiche: Ingenieurswissenschaft, Rechtswissenschaft, } \\
\text { Medizin, Naturwissenschaften, Sozialwissenschaften, Wirt- } \\
\text { schaftswissenschaften (Referenzkategorie: kein Doktortitel) }\end{array}$ \\
\hline Stimmendifferenz & $\begin{array}{l}\text { Differenz zwischen den erzielten Stimmen des Kandidaten und } \\
\text { dem Ergebnis des Gewinners des Mandats }\end{array}$ \\
\hline Anzahl Dr. im Wkr. & $\begin{array}{l}\text { Anzahl der Direktkandidaten im Wahlkreis (Wkr.) mit } \\
\text { Doktortitel }\end{array}$ \\
\hline Dr. (gewichtet) & $\begin{array}{l}\text { Wenn der Direktkandidat keinen Doktortitel führt 0, wenn } \\
\text { der Kandidat einen Doktortitel führt, ist die Variable definiert } \\
\text { als: Anzahl der Direktkandidaten im Wahlkreis geteilt durch } \\
\text { die Anzahl der Direktkandidaten mit Doktortitel (standardi- } \\
\text { siert auf den Wertebereich } 0 \text { bis 1) }\end{array}$ \\
\hline Zweitstimmen (in \%) & $\begin{array}{l}\text { Anteil der Zweitstimmen der Partei des Direktkandidaten in } \\
\text { Prozent }\end{array}$ \\
\hline Parteizugehörigkeit & $\begin{array}{l}\text { CDU/CSU, Die Linke, FDP, Grüne, SPD. Referenzkategorie: } \\
\text { Sonstige enthält Bayernpartei (BP), Bürgerrechtsbewegung So- } \\
\text { lidarität (BüSo), Die Violetten, Deutsche Kommunistische Par- } \\
\text { tei (DKP), Die Tierschutzpartei, Die Familien-Partei Deutsch- } \\
\text { lands (FAMILIE), Marxistisch-Leninistische Partei } \\
\text { Deutschlands (MLPD), Nationaldemokratische Partei } \\
\text { Deutschlands (NPD), Partei Bibeltreuer Christen (PBC), Pira- } \\
\text { ten Partei, Republikaner (REP), Rentnerinnen und Rentner } \\
\text { Partei (RRP), Volksabstimmung, Ökologisch-Demokratische } \\
\text { Partei (ödp). }\end{array}$ \\
\hline Berufliches Wirken & $\begin{array}{l}\text { Einfache und qualifizierte manuelle Berufe, Techniker / Ingeni- } \\
\text { eure, Einfache / Qual. Dienste, Semi- / Professionen, Einfache / } \\
\text { Qual. Verwaltung / Manager. Referenzkategorie: Nichter- } \\
\text { werbstätige, Studenten, Rentner, Hausfrauen, Agrarberufe }\end{array}$ \\
\hline Geschlecht & Weiblich $(1=\mathrm{ja}$, andernfalls 0$)$ \\
\hline Alter & Alter des Kandidaten in Jahren (logarithmiert) \\
\hline Listenplatz (Dummy) & $\begin{array}{l}\text { Kandidat ist auch auf der Liste seiner Partei vertreten }(1=\mathrm{ja} \text {, } \\
\text { andernfalls } 0)\end{array}$ \\
\hline Mitglied des Bundestags & Kandidat ist bereits $\operatorname{MdB}(1=j a$, andernfalls 0$)$ \\
\hline Ministerposten & $\begin{array}{l}\text { Kandidat war bereits Minister/in im Kabinett Merkel oder } \\
\text { Fraktionsvorsitzende/r im Bundestag }(1=\mathrm{ja} \text {, andernfalls } 0)\end{array}$ \\
\hline
\end{tabular}


Appendix Tabelle 2: Zusammenfassung des Datensatzes

\begin{tabular}{lcccc}
\hline Variable & Mittelwert & Std. Dev. & Min. & Max. \\
\hline Stimmenanteil (\%) & 14,83 & 14,46 & 0,08 & 68,07 \\
Stimmen (absolut) & $21.459,70$ & $21.276,68$ & 118 & 101.261 \\
Dr. & 0,11 & 0,31 & 0 & 1 \\
Prof. & 0,01 & 0,08 & 0 & 1 \\
Dr. Ingenieurswissenschaft & 0,01 & 0,10 & 0 & 1 \\
Dr. Rechtswissenschaft & 0,03 & 0,16 & 0 & 1 \\
Dr. Medizin & 0,02 & 0,12 & 0 & 1 \\
Dr. Naturwissenschaften & 0,01 & 0,11 & 0 & 1 \\
Dr. Sozialwissenschaften & 0,03 & 0,18 & 0 & 1 \\
Dr. Wirtschaftswissenschaften & 0,01 & 0,12 & 0 & 1 \\
Stimmendifferenz (\%) & 27,79 & 16,98 & 1 & 67,967 \\
Stimmendifferenz (absolut) & $38.946,68$ & $24.068,84$ & 1 & 98.979 \\
Anzahl Dr. im Wkr. & 0,72 & 0,88 & 0 & 4 \\
Dr. gewichtete & 0,04 & 0,14 & 0 & 1 \\
Zweitstimmen (\%) & 14,28 & 11,94 & 0,02 & 54,47 \\
Zweitstimmen (absolut) & $20.711,31$ & $17.615,21$ & 41 & 86.686 \\
CDU/CSU & 0,15 & 0,36 & 0 & 1 \\
Die Linke & 0,15 & 0,36 & 0 & 1 \\
FDP & 0,15 & 0,36 & 0 & 1 \\
Grüne & 0,15 & 0,35 & 0 & 1 \\
SPD & 0,15 & 0,36 & 0 & 1 \\
manuelle Berufe & 0,07 & 0,26 & 0 & 1 \\
Techniker / Ingenieure & 0,06 & 0,24 & 0 & 1 \\
Einfache / Qual. Dienste & 0,06 & 0,24 & 0 & 1 \\
Semi- / Professionen & 0,29 & 0,45 & 0 & 1 \\
Einfache / Qual. Verwaltung / Manager & 0,41 & 0,49 & 0 & 1 \\
Weiblich & 0,25 & 0,43 & 0 & 1 \\
Alter in Jahren (ln) & 3,82 & 0,28 & 2,89 & 4,49 \\
Listenplatz (Dummy) & 0,67 & 0,47 & 0 & 1 \\
Mitglied des Bundestags & 0,17 & 0,37 & 0 & 1 \\
Ministerposten & 0,01 & 0,10 & 0 & 1 \\
\hline
\end{tabular}

Anmerkung: $\mathrm{N}=\mathbf{2 0 0 8}$ Kandidaten. Der Datensatz des Bundeswahlleiters beinhaltet 2195 Direktkandidaten, davon waren jedoch 187 Personen sogenannte Einzelbewerbungen ohne Parteizugehörigkeit oder gehörten einer Partei an, die nicht auf der Wahlliste (Zweitstimme) vertreten war. 


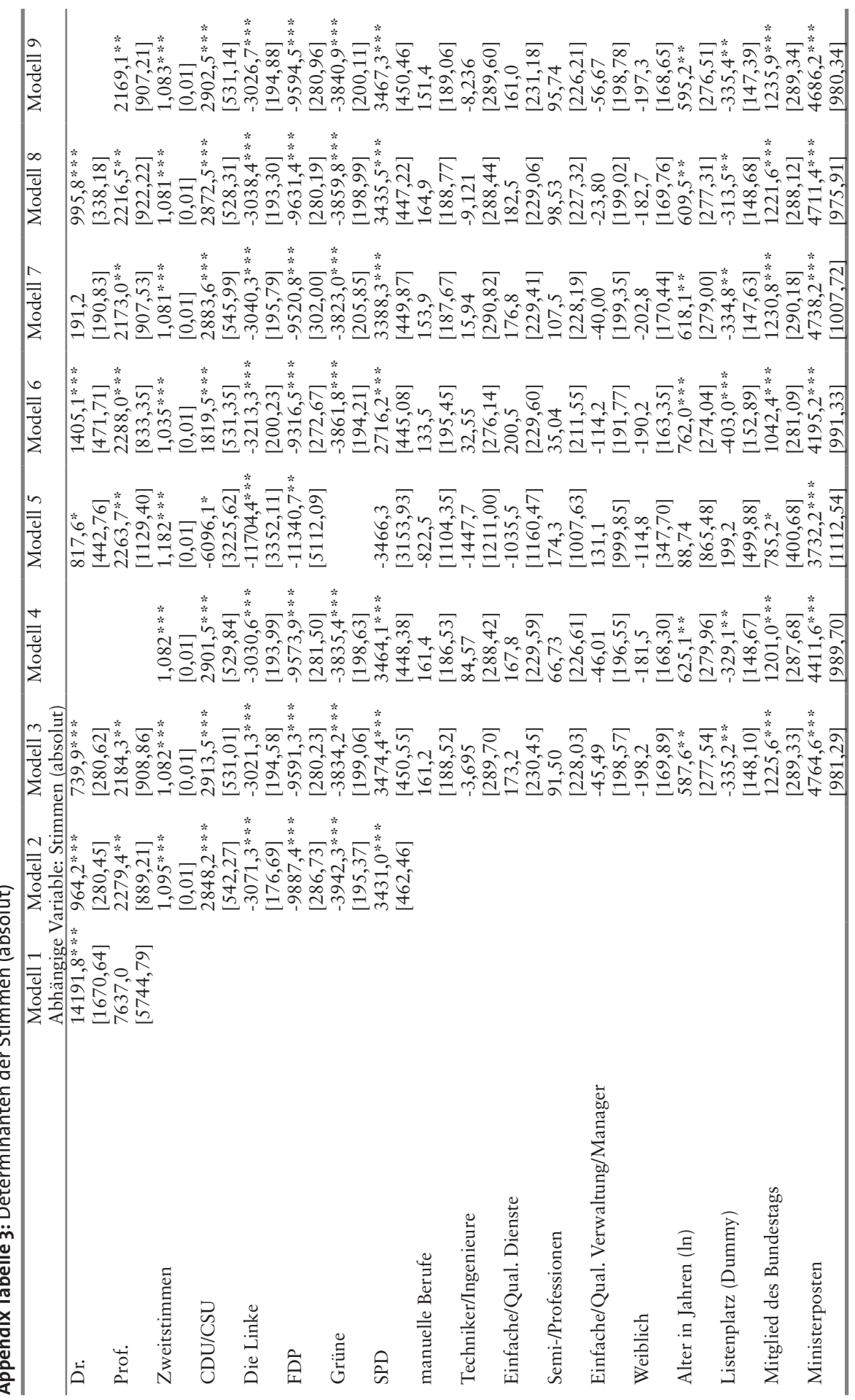




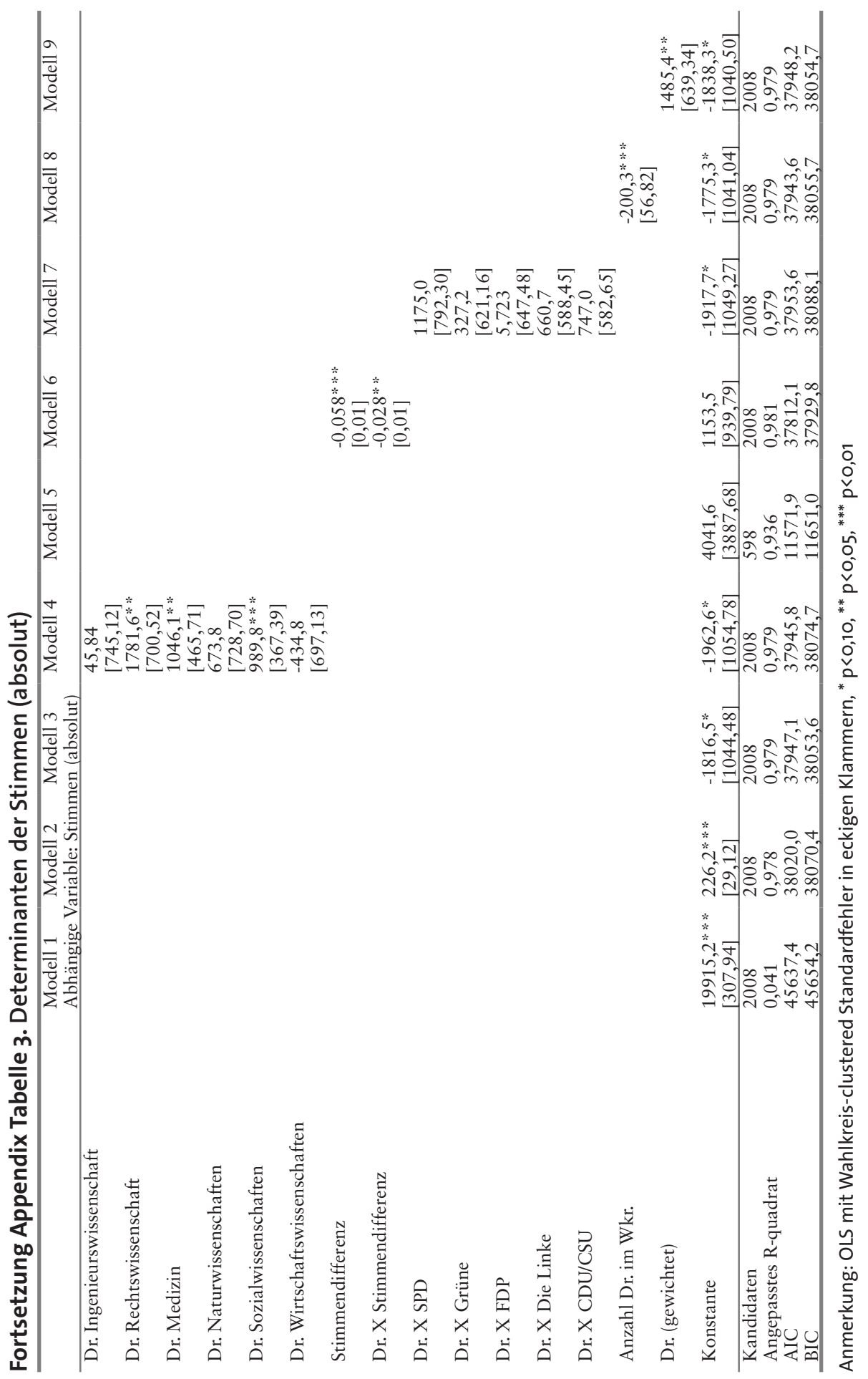

